Delft University of Technology

\title{
Computational modeling of Li-ion batteries
}

Grazioli, Davide; Magri, M.; Salvadori, A.

DOI

10.1007/s00466-016-1325-8

Publication date

2016

Document Version

Accepted author manuscript

Published in

Computational Mechanics

\section{Citation (APA)}

Grazioli, D., Magri, M., \& Salvadori, A. (2016). Computational modeling of Li-ion batteries. Computational Mechanics, 58(6), 889-909. https://doi.org/10.1007/s00466-016-1325-8

\section{Important note}

To cite this publication, please use the final published version (if applicable).

Please check the document version above.

\section{Copyright}

Other than for strictly personal use, it is not permitted to download, forward or distribute the text or part of it, without the consent of the author(s) and/or copyright holder(s), unless the work is under an open content license such as Creative Commons.

Takedown policy

Please contact us and provide details if you believe this document breaches copyrights.

We will remove access to the work immediately and investigate your claim. 
(C) 2018 Manuscript version made available under CC-BY-NC-ND 4.0 license

https://creativecommons.org/licenses/by-nc-nd/4.0/

\title{
Computational modeling of Li-ion batteries
}

\author{
Grazioli D. ${ }^{1,2}$, Magri M. ${ }^{1}$, Salvadori A. ${ }^{1,3}$ \\ ${ }^{1}$ DICATAM - Dipartimento di Ingegneria Civile, Architettura, Territorio, Ambiente e di Matematica \\ Università di Brescia, via Branze 43, 25123 Brescia, Italy \\ ${ }^{2}$ Faculty of Civil Engineering and Geosciences, Delft University of Technology \\ P.O. Box 5048, 2600 GA Delft, The Netherlands \\ ${ }^{3}$ Department of Aerospace \& Mechanical Engineering, University of Notre Dame, \\ 117D Cushing Hall of Engineering, Notre Dame, IN 46556
}

\begin{abstract}
This review focuses on energy storage materials modeling, with particular emphasis on Li-ion batteries. Theoretical and computational analyses not only provide a better understanding of the intimate behavior of actual batteries under operational and extreme conditions, but they may tailor new materials and shape new architectures in a complementary way to experimental approaches. Modeling can therefore play a very valuable role in the design and lifetime prediction of energy storage materials and devices.

Batteries are inherently multi-scale, in space and time. The macro-structural characteristic lengths (the thickness of a single cell, for instance) are order of magnitudes larger than the particles that form the microstructure of the porous electrodes, which in turn are scale-separated from interface layers at which atomistic intercalations occur. Multi-physics modeling concepts, methodologies, and simulations at different scales, as well as scale transition strategies proposed in the recent literature are here revised. Finally, computational challenges toward the next generation of Li-ion batteries are discussed.
\end{abstract}

\section{Introduction}

Greenhouse gas reduction strategies call for developing and deploying innovative technologies. Industrial production of high capacity energy storage devices is one of the major challenges to achieve a low-carbon global economy in the long-term. Li-ion batteries, LIBs, currently have the highest energy storage density of any rechargeable battery technology [1]. The present commercial realities, however, are not yet at such a technological level to meet the requirements of ambitious actions to tackle climate change, as for powering Electric Vehicles (EVs) to displace fossil fuel transport systems.

Since the commercial spreading begun by Sony in 1991 [2], Li-ion battery cells have been widely used to supply electric portable devices such as mobile phones, laptop computers and cameras. Being the latter characterized by limited lifetime, battery aging was not a central topic. The expected use of LIBs for highpower and high-capacity demanding systems, as EVs, $[3]$ and for storage systems for renewable energy sources makes contained capacity fading and power loss nowadays priorities for the world-wide research community. Whereas experimental studies are the backbones of batteries investigation, modeling can provide fundamental contributions, particularly in tailoring material performances and degradation.

Research activities carried out worldwide over the last few years call attention to the multi-scale and multi-physics modeling of storage cells[4] to predict conditions to develop the next generation of batteries for higher capacity and longer cycling life. Computational simulations, based on rigorous theoretical modeling and coupled to validation and quantification of the uncertainties, have the potential to enhance batteries' performances, tailor architectural configurations toward optimal functioning of energy storage devices, and shape new materials for greater capacity and power release. Accurate reviews of battery management systems in electric vehicles [5], of prognostics and health monitoring [6], of prominent phenomena occurring during common operations as well as of the material response to solicitations and of the aging mechanism [7] have 
been recently published. Apparently though, a careful account of the broad literature on computational modeling and simulations appears not to have been considered yet, with a few exceptions [8].

This paper aims at filling this gap, by providing a comprehensive overview and description of computational models and methods proposed in recent years for batteries. Modeling of composite electrodes and electrolytes - either solid or liquid, of core functioning of cells, of intercalation of lithium ions in active materials and their multi physic description, with special emphasis on mechanical behavior and failure will be reviewed and discussed. The main target is to underline the efforts of the scientific community in modeling and simulating Li-ion batteries, by no means presuming to be exhaustive of the widespread literature available on the topic.

Current challenges - including the lack of full 3D multi-scale modeling of the multi-physics processes from atomistic to continuum, of hot spots generation, phase-segregations and mechanical failure - are also identified and approaches to address them are devised.

The paper is organized as follows. Section 2 illustrates basic concepts of Li-ion batteries, highlighting the multi-physics processes that occur at different length and time scales. Sections 3-5 are the core of this review and examine several computational models for the processes of section 2 that have been developed in recent years. Macroscopic models 3, microstructural models 4 as well as multiscale models 5 are separately dealt with.

\section{Processes, modeling, simulations.}

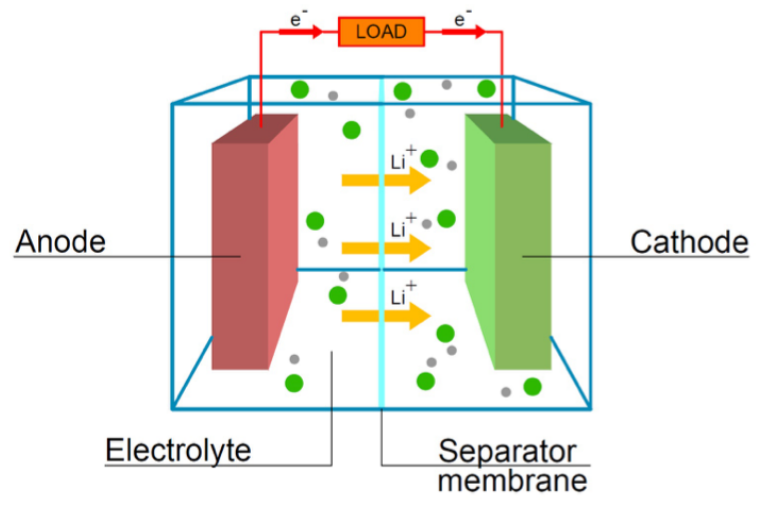

Figure 1: Sketch of a Li-ion battery cell with porous electrodes. Additives are used to create conductive networks in both electrodes, to increase the electronic conductivity. Additives include large (graphite) and small (carbon black) conductive particles, which are bound to the active particles that host Lithium by a polymer binder.

An electrochemical cell consists of two electrodes, a separator and, for liquid electrolytes, an electrolytic solution. Electrons flow externally and through the electrodes, facilitated by a network of conductive particles. Mass and positive charges are transported in form of ions through the electrolyte from one electrode to the other, to bring reactants to the interfaces where intercalation reactions occur. A sketch of a LIB cell is depicted in Figure 1, whereas Figure 2 illustrates the main processes that take place during operations. As shown in Figure 3, each process requires its own modeling.

As already pointed out [9], the term battery modeling is shared by different approaches, each of which makes use of its own methodology to achieve specific targets. Some models for instance define the battery as a black box, aiming at empirically describing its system-level characteristics, such as capacity, efficiency, and voltage. Peukert's law, which correlates the rate of discharge and the capacity, falls into this class $[10,11]$. Other mathematical models study the non-linear capacity/recovery effects in LIBs in a similar 
(1)

(2)

Intercalation reactions

Electronic conduction

3 Diffusion in active particles

(4) Swelling, stress generation, failure

(5) Phase segregation

(6) Migration

(7) Electrolysis
Ionic diffusion in the electrolyte

Ionization reaction / incomplete dissociation

(10) SEl formation/disruption

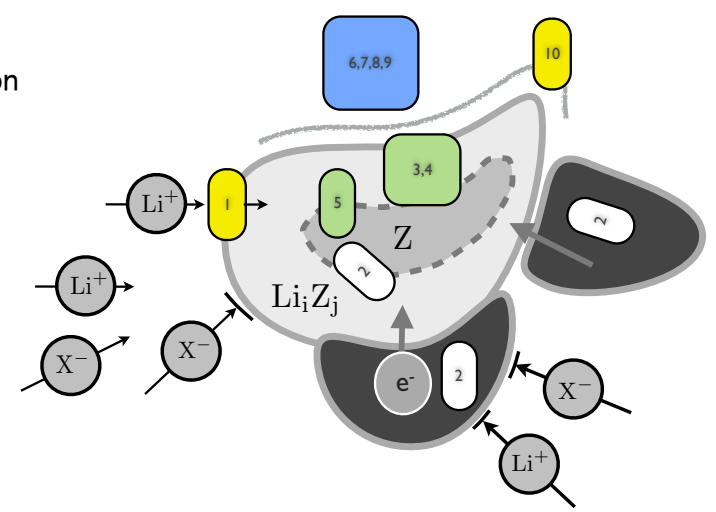

Figure 2: A list of processes that take place in a battery during normal operation.

(1) Butler-Volmer equation Gouy-Chapman theory

(2) Ohm's law

3 Mass balance equations

(4) Principle of virtual power

4 TD consistent constitutive models

(5) Sharp interface modeling Cahn-Hilliard theory

Electroneutrality -

6) Maxwell's equation in electro-quasi-statics

7 Faraday's law
Mass balance equations

Reaction rate in mass balance equations

(10) SEl formation/disruption models

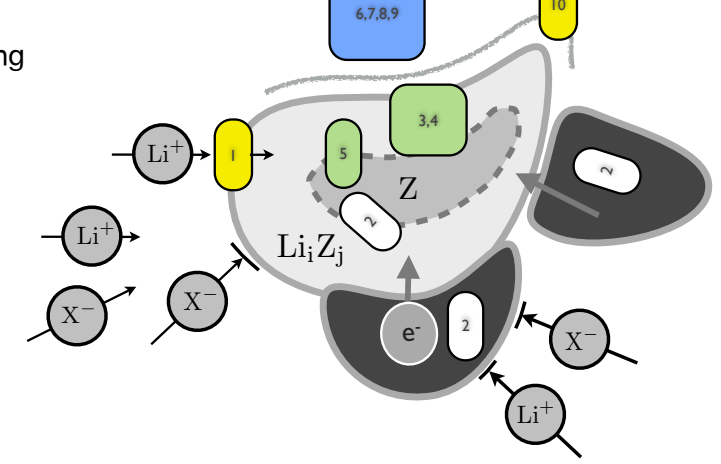

Figure 3: A list of models for the processes that take place in a battery during normal operation.

way[12, 13, 14]. Equivalent circuit models simulate the battery with combination of variable voltage sources, resistors, and capacitors[15, 16]. Several of these models have been developed in the literature[17], including Thevenin equivalent circuit models [18], impedance-based models [19], and runtime-based models [20]. A recent review can be found in [21]. 
Although those models have been quoted here for the sake of completeness, the present review focuses on the multi-scale and multi-physics modeling of the processes that take place during charge/discharge, from the atomistic size up to the cell size. This choice has a sound motivation, since theoretical and computational modeling not only provide the ability of understanding the microscopic behavior of batteries under operational and extreme conditions, but they may also be tailored to devise and shape new materials as well as new architectures in a way complementary to the experimental approaches. Modeling of multiscale and multi-physics processes taking place during charge/discharge can therefore play the most valuable role for battery design and lifetime prediction.

Any rigorous model of physical phenomena stems from a few pillars. They are, in order: the balance (or continuity) equations; the thermodynamic analysis, in terms of energy and entropy balance; the constitutive theory and specifications; in the presence of large deformations, objectivity shall also be properly investigated. Governing equations result from this fundamental sequence of tasks. Scientific rigor claims that this sequence shall be respected, but this is unfortunately not always the case in the literature on battery modeling. In particular, from the second law of thermodynamics restrictions arise that constitutive modeling should account for $[22,23,24,25,26]$. Furthermore, in multi-scale approaches, the so called Hill-Mandel condition must be considered, to guarantee that energy is neither artificially created nor annihilated in the scale transitions.

Despite many progresses have been made in the last decades, modeling the complex microstructure of a battery still represents an open challenge. The different nature of the phenomena involved (mechanical, electrical, electrochemical, and thermal) and the interactions among them lead to complex mathematics with a very high number of unknown fields (displacements, electric potential, concentrations, temperature). Moreover all the fields are coupled through non-linear constitutive relations, thus leading to non linear partial differential equations that require iterative algorithms to be numerically solved. As a further complexity in order to achieve predictive capabilities, several parameters usually must be calibrated. Uncertainties in the measurement of those parameters are significantly high for $\operatorname{LIBs}[27,28]$.

Mass transfer entails a description of the movement of mobile ionic species. In the absence of convection, as usually assumed under operation conditions, movement of species is governed by diffusion, driven by gradients of concentration, or by migration, driven by an electric field. The intercalation reactions taking place at the electrode-electrolyte interfaces, can be either described by Butler-Volmer-type equations or by more complex analysis $[29,30,9]$ of the phenomena that occur in the atomistic-size layers, termed after Stern and Gouy-Chapman.

Both negative - carbonaceous, mainly graphite, (C) - and positive - $\mathrm{LiCoO}_{2}(\mathrm{LCO}), \mathrm{LiNiO}_{2}(\mathrm{LNO})$, $\mathrm{LiFePO}_{4}$ (LFP), and $\mathrm{LiMn}_{2} \mathrm{O}_{4}$ (LMO) - electrodes intercalation materials exhibit phase transition [31, 32]. In some cases the transition is sharp (e.g. from crystalline to amorphous in Silicon) and can be clearly defined by an atomically thick boundary [33] where a chemical reaction takes place. The material properties may change among phases, thus leading to unexpected stress scenario which are under intense study. The reaction rate at the front has been detected as one of the limiting factors for the electrode charge/discharge rate.

Modeling power loss and capacity fade with electrochemical cycling - either in operating or non operating conditions - is becoming a major branch of research $[34,35,36,37,38]$, especially in connection with high-power and high-density cells that are prone to rapid degradation. The main mechanisms of aging can be categorized in four groups, namely: surface film formation (solid electrolyte interphase (SEI), Lithium plating), bulk changes (phase segregation), mechanical effects due to lithiation (fracturing, dissipation, grinding), and parasitic reactions (corrosion, binder degradation). An up to date review of the literature on aging of Lithium-ion batteries for electric vehicles can be found in [3], with special emphasis on physicochemical transformations. Recent publications on aging and degradation accentuated the role of mechanical detrimental effects. Although individual mechanism strongly depend on the materials in the cell, general effects can be pointed out. Phase-segregation and large volume changes in the active particles are associated with the intercalation of neutral $\mathrm{Li}$ in the hosting storage materials. Swelling induce inelastic effects, micro-cracks and particle fracture, decrepitation or pulverization, loss of integrity and loss of electric contact with the current collector, finally leading electrodes to die. 
Functional materials for energy conversion and storage exhibit strong coupling between electrochemistry and mechanics $[39,40]$. Volume expansion and fracturing of composite electrode components may occur during battery standard operations. Active materials in composite electrodes experience swelling and shrinking during cycling due to Li-ions insertion and extraction. Volume changes reported in the literature range between $6.5 \%$ for LMO [41] and about $10 \%$ for carbonaceous materials [42, 43]. Such an amount of deformation may induce stress which in turn cause fracture and detachment of particles from the conductive network. In this regard, experimental data have been reported for various active materials:, such as LCO [44], LMO $[45,46]$, LFP $[47,48]$ and C $[49,50,43,51]$. The problem is even more relevant in materials with large storage capacity materials. For instance, the tremendous volume change experienced by Li-alloys, e.g., up to $300 \%$ for Li-Si [52] causes fracturing after a small number of cycles.

Deformations in composite electrodes may cause contact loss among their different components accompanied by irregular SEI growth that can modify the porosity of the solid matrix and reduce the overall ionic conductivity.

Temperature influences drastically the performance of batteries under operation conditions. In extreme conditions, temperature increase can initiate a sequence of detrimental effects, known as thermal runaway, that may lead to a destructive result. Three paradigmatic mechanisms for thermal runaway have been recently either experimentally discovered or numerically envisaged. The first mechanism [34, 53] relates to deposition of metallic Lithium during charging, which may compromise cell safety because of dendritic growth and internal short-circuiting. The deposition reaction occurs upon overcharge, fast charge, or at low temperature. Thermally induced mechanisms have been experimentally investigated in [54] in the presence of an external heat source, as for the failure of neighboring cells. They are fueled by the continuous exothermic decomposition and reformation of the solid electrolyte interphase layer at negative electrode/electrolyte interface, inducing a complex chain of events up to battery explosion. Drops in concentrations at the same location, which have been predicted in the presence of fast charge/discharge processes [55, 56], can trigger a similar series of events. Modeling and simulation of those mechanisms involve complex physical processes coupled across a wide range of length and time scales.

\section{Macroscopic models}

Although multi-physics phenomena described in figure 2 take place at the length scale of the electrode particles - namely three order of magnitudes below the battery cell size - or even at smaller scales, boundary conditions that drive the response of batteries cannot be identified from experimental observations at those scales. Boundary conditions are more naturally defined at the battery cell scale, and they depend upon the process (galvanostatic/potentiostatic, charge/discharge) and upon the geometry of the cell (cylindrical, pouch, others). The former remark, together with the quest of feasibility of numerical simulations for the models described in figure 3 at the finest scale with the state of the art of high performance computing, lead to the conclusion that the computational modeling of batteries must unavoidably be treated via multi-scale approaches. Nevertheless, a large amount of research in batteries focused on one-dimensional, single scale modeling, accounting in the best possible way for the underlying microstructure.

\subsection{Macroscopic models for liquid electrolytes and separators}

In liquid-electrolyte batteries, the inter-electrode medium is a composite structure formed by the electrolyte and a separator membrane, that includes a network of interconnected and irregular pores. Most liquid electrolyte models concern a single binary electrolyte, i.e. a solution of a binary salt, say LiX where X can for instance [59] be $\mathrm{PF}_{6}$, plus a solvent in which the ionic concentration varies in the cell. Mass transfer in an electrolytic solution requires a description of the motion of mobile ionic species $\mathrm{Li}^{+}$and $\mathrm{X}^{-}$which is due to diffusion, migration, and advection. Even though the latter contribution might be relevant for some electrochemical systems [60,61], especially under abuse or extreme conditions [62], advection is usually neglected in LIBs models.

In many cases, the ionic transport is described assuming the electrolyte as an ideal infinitely diluted solution. Under this hypothesis the energetic interactions between different species are neglected; hence, the flux of a species is proportional to the gradient of its own electrochemical potential [63]. For systems involving $n$ different species, the set of $n$ mass balance equations contains $n+1$ unknowns, typically $n$ 

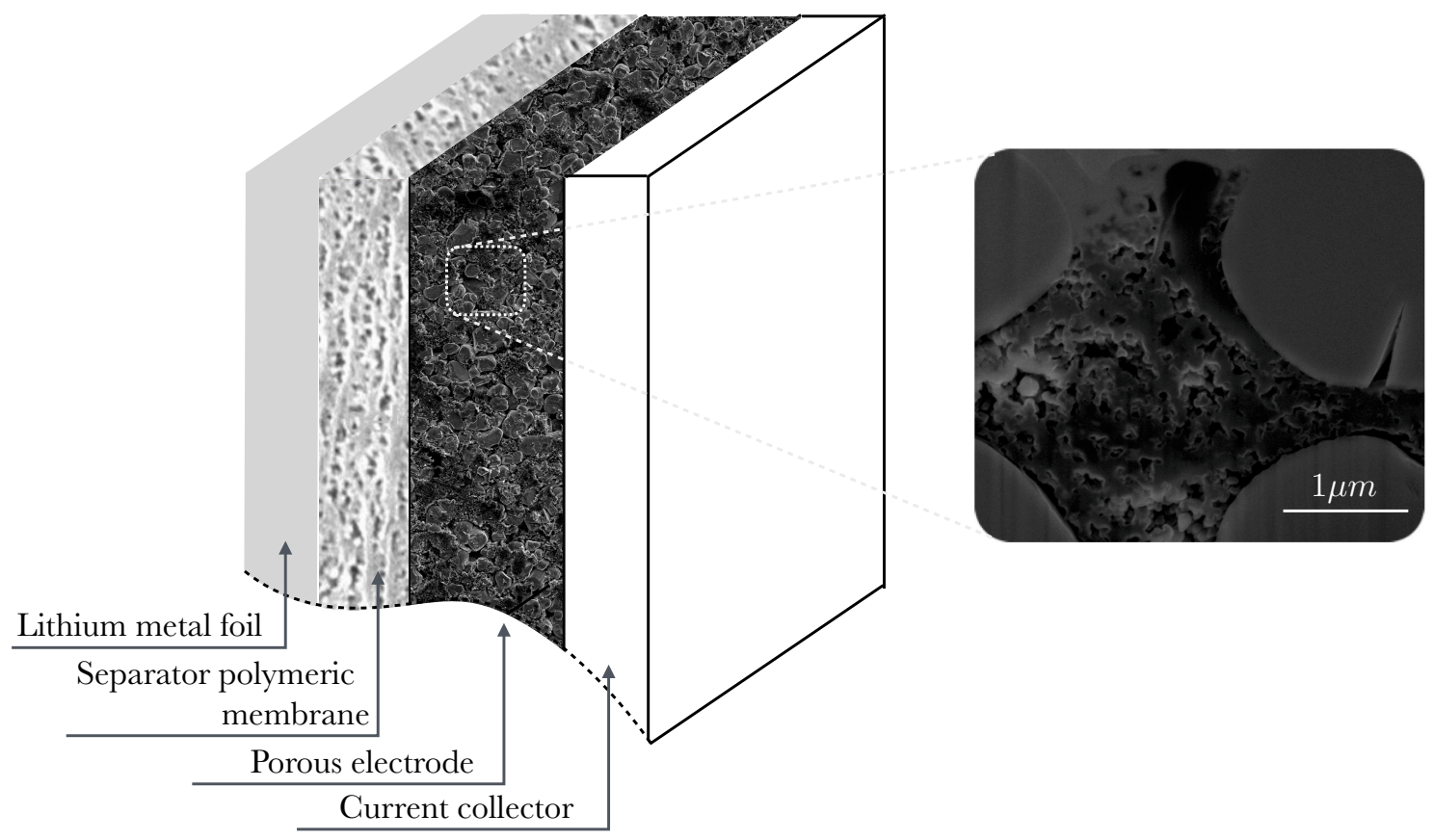

Figure 4: Sketch of a Lithium metal/liquid electrolyte separator/porous insertion electrode cell and a FIBSEM image of a $\mathrm{LiCoO}_{2}$ commercial electrode. Large active particles are clearly visible, together with the carbon additive particles that form a micro-porous structure between them. Separator scanning electrode microscope images can be found for instance in [57, 58].

mass concentrations plus the electric potential. An additional equation is mandatory and the most common selection in battery modeling is the electroneutrality condition [64].

Electroneutrality can be defined as follows: over macroscopic distances the difference in concentrations of the ionic species is small compared to the ionic concentrations [65] and can be neglected. Such an assumption is valid at material points "far" from the electrode/electrolyte interfaces, where high electric fields separate positive and negative charges across very narrow layers.

Noteworthy, electroneutrality is not a fundamental law, but rather an approximation towards the solution, which can lead to paradoxes if not consciously adopted. Dickinson et al. [66] have shown that electroneutrality does not constrain in any way the electric field to satisfy Maxwell's equations. Danilov and Notten [63], while discussing numerical simulations stemming from the electroneutrality assumption, pointed out an unjustified electric field in spite a good estimation of the ionic concentration. More general formulations that do not account for electroneutrality in the set of balance equations are required in multi-scale approaches $[67,68,55,56]$.Their description is thus postponed to section 5 .

Separators have to be designed to prevent internal short circuiting while providing a path for ionic conduction in the liquid electrolyte throughout its open porous structure. Examples of separators are microporous polymer membranes, non-woven fabric mats, and inorganic composite membranes. Although the separator does not participate in the electrochemical reaction it influences the performance of Li-ion battery cells, as experimentally observed by Djian et al. [69].

Morphological features are macroscopically accounted for through global parameters, such as porosity and tortuosity. The former quantifies volume fraction occupied by the pores; the identification of an optimal value is a compromise between the minimization of the ionic resistance, enabling high-specific battery power (high porosity and large mean pore size), and the limitations dictated by the mechanical resistance of the membrane, preventing the risk of inner battery electrical shorting (thick membrane and low porosity). The tortuosity is a measure of how the conductive pathways deviate from an ideal condition of straight channel of uniform cross section. Increasing tortuosity also increases the mean path length of ions, eventually favoring undesired effects [70]. Many attempts can be found in the literature [71, 72, 73, 74, 75] to relate 
porosity and tortuosity to effective conductivity and diffusivity of inhomogeneous media. The influence of the underlying microstructure onto macroscopic material properties is a goal of homogenization theory, which will be discussed in section 5 .

As the integrity of the separator is crucial to the performance and safety of batteries, stress analyses for the separator can be found in literature [58, 76], taking also into account the SEI layer and its growth as one of the main degradation mechanisms [77].

\subsection{Macroscopic models for solid electrolytes}

Solid electrolytes are of increasing interest due to their possible application in power microelectronic devices, such as micro-electomechanical systems (MEMS), remote sensors, self powered integrated circuits and health care implants $[78,79,80]$. Despite a generally limited ionic conductivity, up to three orders of magnitude smaller than conventional liquid electrolytes [81], their application is characterized by undeniable advantages: safety, high energy density due to usage of a pure Lithium metal anode is enabled yet ensuring stability, no need for casting, no leakage, resistance to shock and vibration, resistance to pressure and temperature variations, electrochemical stability $[82,83,81,84]$.

Inorganic glasses, with particular reference to lithium phosphorus oxynitride (LiPON), find application in many solid state thin-film lithium batteries[85]. A widespread example is the $\mathrm{Li} / \mathrm{LiPON} / \mathrm{LiCoO}_{2}$ system, investigated by Fabre et al.[83] and Danilov et al.[86]. LiPON solid electrolyte is a glass-forming system in which immobile, oxygen-binded Lithium is transferred to mobile Lithium by means of a ionization reaction, during which a negative charge is released. This process was described by Danilov et al., who made use of the Nernst-Plank equation and the electroneutrality assumption to describe mass transport of Li-ion and negative charges during a battery subjected to a discharge process. A simplified approach was pursued by Fabre et al., who assumed the ionic concentration to be uniform in the bulk electrolyte during both charge and discharge processes. The mobile charges being exclusively Li-ions, Ohm's law was used to relate the current density to the electric potential distribution across the LiPON. The effect of temperature on the input parameters of the model was also studied.

Polymer electrolyte find application in three-dimensional [78] and flexible [87] batteries. Ionic transport in these materials is related to the segmental motion of the polymer chains. The repeated association and dissociation of the ions with the polymer segments and the continuous rearrangement of the latter allow ions to be transferred from one electrode to the other [88]. As the motion of the polymer chains is enhanced at temperatures above the glass transition temperature, higher ionic conduction are also observed at higher temperatures [82]. For reviews on the ionic transport mechanisms in polymer electrolytes, their dependence on temperature and modeling approaches at different scales the reader is referred to [89, 90].

Solid electrolyte have recently modeled from a continuum level perspective by Natsiavas et al. [91]. The latter investigated the effect of pre-stresses on the dendrites formation of lithium metal anode either in contact with LiPON electrolyte or dioxolane-dimethoxy ethane, a soft polymer. In both cases the electrolyte was modeled as a linear elastic material undergoing small deformations. A continuum model for diffusion of multiple charged species in a solid medium in the presence of stress, electrostatic and chemical potential gradient was developed by Bucci et al. [92]. The model, applied for the investigation of the effect of mechanical stress in kinetic demixing and ambipolar diffusion (both phenomena are peculiar of solid oxide fuel cell) is also amenable for solid electrolyte modeling.

\subsection{Macroscopic models for porous electrodes}

For porous heterogeneous electrodes, one-dimensional macroscopic mathematical models reflect reality only in an average sense, that must be properly defined. Porous electrodes are in fact multi-phase structures, which include a network of interconnected and irregular pores and channels [58]. They consist of active particles bound by a polymer to a network of conducting particles, see Figure 4. Storage materials are subjected to Lithium intercalation, while conducting particles provide a conductive path that enhances the electronic mobility from the current collector to the active particles surface. Lithium transport in form of ions takes place in the electrolytic solution. The porous configuration increases the effective interfacial area per unit volume between the electrolyte and the active material, favoring the intercalation of Lithium. The rate at which the latter occurs depends on the structure and on the material properties of the phases of the 
composite electrodes [64]. These factors influence the potential drop and concentration changes in both the solution and solid phases [93].

From the seminal works by Newman and coworkers [94, 95] a multitude of models based on the socalled porous electrode theory have been applied to the insertion electrodes. The approach circumvents the description of the complex microstructural geometry and processes, by considering macroscopic averaged quantities "over a region of the electrode small with respect to the overall dimensions but large compared to the pore structure" [64]. Two components, occupying different domains, are considered: an homogeneous separator - in which only the electrolytic solution is present - and multi-component porous electrodes - in which the electrolytic solution and the solid matrix are treated as superposed continua, ensuring connectivity between all points of the electrode [96] - both present at any point of the domain.

The model entails a set of partial differential equations, which describe the processes at the cell scale in an averaged, phenomenological way. The unknown fields are: the molar concentration $c_{i}$ of species $i$ (both in the separator and in the porous electrodes); the electric potentials $\phi_{e}$ and $\phi_{s}$ of the electrolyte and of the solid phase respectively. The fields are assumed to be continuous functions in time and space. Porosity $\epsilon$ accounts for the void volume fraction, filled by the electrolytic solution. It equals unity in the separator and is strictly smaller than one in the porous electrodes [97, 98].

Mass conservation equation

$$
\frac{\partial \epsilon c_{i}}{\partial t}+\operatorname{div}\left[\vec{h}_{i}\right]=R_{i}
$$

is enforced to any mobile species $i$ in the electrolytic solution. It relates variations of the molar concentration to the molar mass flux $\vec{h}_{i}$ and to the mass supply $R_{i}$. The latter is identically equal to zero in the separator (unless chemical reactions are explicitly considered) while it macroscopically accounts for the microscopic pore-wall flux density in the porous electrodes [64].

The current $\vec{i}$ is defined as the charge flux density per unit area. It coincides with the electrolytic current $\vec{i}_{e}$ within the separator where the ions are the only mobile charged species, and with the sum $\vec{i}_{e}+\vec{i}_{s}$ in the porous electrodes. Charge conservation is ensured being $\vec{i}_{s}$ the amount of current carried by the electrons in solid phase within porous electrodes.

By imposing the electroneutrality condition

$$
\sum_{i} z_{i} c_{i}=0
$$

where $z_{i}$ is the charge number of species $i$, the net charge is always zero and the total current density satisfies the charge balance equation

$$
\operatorname{div}[\vec{i}]=0
$$

both in the separator and in the porous electrodes.

Constitutive assumptions relate the mass fluxes $\vec{h}_{i}$, the current densities $\vec{i}_{e}$ and $\vec{i}_{s}$ and the bulk terms $R_{i}$ to the molar concentrations $c_{i}$ and the electric potentials $\phi_{e}$ and $\phi_{s}$, which are the thermodynamic variables and the unknown fields of the model. Faraday's law relates the electrolytic current density to the ionic mass fluxes in the electrolyte, whereas Ohm's law is assumed to govern the movement of electrons in the matrix phase. Either dilute, moderately-dilute or concentrated solution theories may be adopted, leading to thermodynamic scenarios characterized by an increasing degree of mathematical complexity. For the sake of brevity, the reader is addressed to specific literature $[63,97,98,99,64,100]$ for the explicit expressions of the constitutive equations.

The macroscopic physical properties that enter the constitutive equations assume the meaning of averaged micro-structural features within the porous electrode. For example, the effective ionic diffusivity in random porous structures intrinsically accounts for the porosity and the for the deviation of paths within pores from being straight channels (tortuosity) [75].

A Butler-Volmer type kinetic expression was introduced to account for the kinetics of the charge-transfer processes at the electrode in place of the assumption of infinitely fast insertion [100, 97]

$$
i_{n}=i_{0}\left[\exp \left(\frac{\alpha_{a} F \eta}{R T}\right)-\exp \left(-\frac{\alpha_{c} F \eta}{R T}\right)\right]
$$


$i_{0}$ is the exchange current density, whose value varies with the concentrations of reactants and products, temperature, and the nature of the electrode-electrolyte interface; $\alpha_{a}$ and $\alpha_{c}$, called apparent transfer coefficients, express how an applied potential favors one direction of reaction over the other (their values range in literature between 0.2 and 2); $\eta=\phi_{s}-\phi_{e}-U$ is the surface overpotential and $U$ is the open-circuit potential, corresponding to thermodynamic equilibrium conditions (zero net transfer current density). The mass supply $R_{i}$ is related to the average transfer current density $i_{n}$.

The open-circuit potential $U$ is related to the state of charge. The so-called pseudo-2D models[101, 102] have been introduced to investigate such a dependency. The etymology of this class of models arises from the two "unrelated" dimensions that are involved, namely the cell and the active-particles. The latter have been assumed as spherical (with rather few exceptions, e.g. $[103,100]$ ) of constant radius upon intercalation of external species.

Porous electrode theory has been applied with an increasing degree of complexity over time, since enhanced available computational tools became available. In particular, modeling of electrodes attempted to account for the fine microstructure within the porous electrode theory, with strategies sometimes formulated on a phenomenological rather than a solid theoretical ground.

The modeling of full-cells composed by Lithium metal/solid polymer separator/insertion positive electrode and simulation of galvanostatic charge/discharge processes pursued by Doyle et al. [97] enhanced previous approaches $[103,100]$, accounting for interactions of the battery components. Concentrated solution theory was used to describe transport process occurring in the electrolyte.

The "constitutive" relation between the open-circuit potential and the state of charge controls the current distribution inside the porous electrode. Materials characterized by significant changes in open circuit potential for limited variation of the state of charge (e.g. carbonaceous materials) lead to more uniform current profiles in the porous electrode compared to materials exhibiting this dependence to a limited extent (e.g. LMO),[98] and hence to a better utilization [34].

Intercalation materials were regarded as perfectly conductive and interested by Fickian solid diffusion, as in $103,100,97,96]$. Constant physical properties were used for all the components. Film resistances on both electrodes was introduced by Doyle et al. [104].

All the models described above share some fundamental hypotheses. The electrolyte consists of a binary salt in a single solvent [100, 97, 96, 98], a picture recently questioned under high C-rates [105, 106]. Side reactions (e.g. SEI layer formation and electrolyte decomposition) have not been considered, therefore the amount of Lithium during all processes is conserved, which contrasts experimental observations [34]. Volume changes due to intercalation have not been accounted for, therefore the models do not account for mechanical effects that strongly contribute to degradation [107].

Numerical simulations with the porous electrode theory have been carried over a wide range of materials and configurations [97, 98, 108, 104, 105, 109, 110]. Outcomes, in terms of concentration profiles and pore wall flux distribution within composite electrodes during charge/discharge processes, provided meaningful insights on battery design and optimization. As observed by Doyle et al. [97], cell optimization relates to battery configuration, energy and power density requirements of the system, and cost of components. Whereas thinner electrodes limit electrolyte depletion and allow for higher specific power, thicker electrodes increase specific energy by reducing the number of cells in the battery stack and the volume fraction of inactive components [105].

The robustness of the Pseudo-2D approach is assessed by the number of recent models that stem from it, as $[101,111,112]$. Pseudo-2D models also present limitations: typically, the large amount of material parameters requires remarkable experimental efforts. The main drawback though relates to microscopic approximations. Pseudo-2D models do not allow to describe in detail the processes taking place at the finest scale, which can have a significant impact on the overall battery response [99]. The capacity fade due to active particles fracturing and decrepitation, the porosity change caused by SEI formation or reaction products deposition have already been remarked. A thorough discussion on the applicability of macroscopically homogeneous models to Lithium-ion battery description can be found in [113], where a poor predictive capacity is highlighted for high C-rates.

Macroscopic battery models that do not make use of Newman's porous electrode theory have been published, too. In general, they replace the porous structure with aggregates of solid-phase particles, which 
are directly embedded in the electrolytic phase $[114,115,116]$. Mechanical stress generation was in few cases accounted for in the solid-phases [99, 116].

Despite the formulation was derived in $3 \mathrm{D}$, the numerical implementation was often restricted to $2 \mathrm{D}$ [99, 114, 116]. Three-dimensional simulations were developed in [115], where a half-cell Lithium battery was modeled on a LMO spherical particles array. Numerical analyses suggest that small sized particles and regular arrangement ensure higher capacity, especially when active materials are characterized by low diffusivity.

Macroscopic models for solid (non porous) electrodes have also been studied. The geometry of the cell is idealized as blocks stacked in order to reproduce the sequence of the battery components, idealizing the battery with a one-dimensional structure $[30,86,83]$.

\section{Microscopic phenomena and their modeling}

Several models have been proposed to investigate the microscopic response of electrodes during intercalation, in order to provide design criteria for enhancing electrodes performance. In fact, the response of the electrodes upon Lithium uptake and release during batteries charge and discharge depends on the thermochemo-mechanical properties of the compound of active and conductive particles. The micro-structural composition and the geometrical configuration are of paramount relevance for the intercalation process and the performances of the electrodes.

Since the cell is first assembled, charges are localized at the interfaces between electrodes and electrolyte, causing an intense electric field to develop in an adsorption layer (named after Stern [117]) with atomicscale dimensions adjacent to the interface, together with a more diffuse region of charge (known as the Gouy-Chapman layer $[118,119]$ ) in the electrolyte [120]. These regions together (in the order of 10 to 20nm according to the literature [64]) define a so-called electric double layer. An exhaustive dissertation about the electric double layer can be found in classical books $[29,64]$ as well as in more recent literature, among which Bazant and co-workers publications deserve special merit [65, 121, 122].

In Li-ion battery modeling literature, the double layer is generally assumed as infinitesimally narrow, with a few exceptions [123]. Local electroneutrality is generally assumed in the electrolyte and a discontinuity in the potential across the electrode/electrolyte boundary is allowed for (see among others $[60,61,63,116,112]$ ). Butler-Volmer equation $[124,125,126]$ is used to relate the intercalation flux to the potential discontinuity between the electrode and the points in the solution immediately beyond the ideally narrow double layer [127, 128, 129, 99, 116]. Streeter and Compton [130] critically discuss the electroneutrality and the negligibly small double layer approximations, arguing that they are appropriate only if the active particles of the electrode are much larger than the electric double layer. Dreyer et al [131] formulated a continuum model of the layer, including solvation effects of the dissociated ions, as well as the mechanical effects. Landstorfer and Jacob [9] discuss weak and strong electroneutrality conditions and split the electrochemical intercalationdeintercalation process in three simple reactions, in order to define appropriate boundary conditions. That paper also accounts for a large bibliography on the mathematical modeling of intercalation, to which we further address the interested reader.

An accurate morphology reconstruction is a crucial information for micro-scale analysis. This statement applies to localized phenomena, like hot spot formation, as well as to ensemble averaged material properties, which have been shown to be extremely sensitive to the size, shape, and particle distribution within a Representative Volume Element [132].

Accurate reconstructions of the connectivity and of the internal structure of electrodes is a very hard task: binder, active, and conductive particles give rise to tangled and intricated geometries. The complexity of the morphology of electrodes and separator restricted the focus of most publications to the behavior of a single particle, disregarding the influence of the electrode microstructure $[133,134,135,136,137,138,139$, $140,141,142,143,144]$.

The microscopic arrangement of active particles, carbon, binder and pores can be recovered from statistical analyses of 2D cross sections of the electrode achieved through scanning electron microscope with focused ion beam. The particle geometry can either be a simplified abstraction of reality - for example using 
cluster of spherical [74, 145] or ellipsoidal [146] particles that yet represent challenges for computational discretization [147, 148] - or be digital reconstruction of realistic morphology [149, 150, 151, 152, 153, 154, 155] - generally restricted to single-particle analyses.

In recent years major progresses have been made in the area of X-ray computed tomography $[156,157$, $158,159]$ and focused ion beam scanning electron microscopy [149, 160, 151, 161, 162, 163], paving the way to numerical simulations on realistic three dimensional microstructures. They involve high computational costs $[151,147,148]$ and are feasible only in a high performance computing environment.

Modeling temperature evolution in Li-ion battery cells is important in operation conditions $[9,164,165$, $166,111]$, to dictate parameters for cooling devices design, but even more under abuse or extreme conditions, to predict and control thermal runaway $[54,167]$. A recent review can be found in [168].

Whereas operating temperature ranges may vary upon different applications and related national standards, it is generally established that an increase of temperature, either because of external conditions or during fast charge/discharge [69], boosts degradation mechanisms. In general, temperature affects ion mobilities, SEI formation and dissolution, interface reactions - eventually promoting undesired side effects. Accounting for the influence of temperature in modeling is extremely complex mostly because: i) temperature affects transport properties of materials, interface kinetics, electrical and mechanical properties in ways that are difficult to capture experimentally and reproduce numerically [169], even though the general thermodynamic framework that lays the ground of every rigorous theory is nowadays well established [170]; ii) numerical treatment of multi-physics problems in coupled chemo-electro-thermo-mechanics are not completely understood [171]; ii) temperature in the cell may increase after the formation of very localized hot-spots, which are related to the speed of charge/discharge [152].

A few attempts only accounted for realistic microstructure of porous electrodes considering the temperature field and the hot spots formation under extreme conditions in a multi-scale and multi-physics framework $[170,62,153]$.

Notwithstanding the large number of publications focused on Lithium-ion battery, the modeling of the detrimental effects related to aging mechanisms is a relatively recent topic[172]. Arora et al. [34], browsing the most relevant capacity fade mechanisms concerned with side reactions in Li-ion batteries, observed that the only model on the topic available at that time was provided in Darling and Newman [173]. The latter represents the first attempt of simulating aging process in a physical model, as recently remarked also by Barré et al. [7].

Aging affects electrolyte, composite electrodes, as well as the electrode/electrolyte interfaces. Many different factors concur to capacity decrease and power fading of Lithium-ion batteries both during storage and charge/discharge cycling. Calendar aging is monitored in terms of capacity loss, impedance rise, state of charge and state of health [37]; it has been proved to be sensible to storage conditions, especially temperature and state of charge [7]. Cycle aging may be detected through capacity fade, impedance rise and overpotential that can be measured during cycling [37]. Various studies have experimentally shown that it is enhanced by larger amount of charge variation during cycles [174, 175], high charging/discharging voltage [176] and current peaks [7]. Calendar and cycle aging coexist during the whole battery lifetime. An example of estimation and comparison between their relevance on the performance of a C/LFP cell can be found in Safari and Delacourt [177].

The processes by which Lithium is either produced or consumed within a battery cell include Lithium deposition, electrolyte decomposition, active material dissolution, phase changes in the insertion electrode materials, and passive film formation over the electrode and current collector surfaces. Often they are collectively termed side reactions [34]. The interaction among any of the processes mentioned above is favored by the similar timescales over which they occur, making difficult their specific identification. Moreover most of them are strongly related to the peculiar features of the cell (shape, microstructure, material components).

Detrimental effects may also descend from other processes, such as interaction between binder polymer with active and conductive particles, current collector corrosion and plating, gas formation within the cell $[7,3,37]$.

The most relevant aging mechanism for carbon anodes is attributed to phenomena occurring at the electrode/electrolyte interface, where a passivating layer, termed solid electrolyte interface (SEI), prevents further interfacial reactions yet allowing the Lithium ion migration [31, 178]. Notwithstanding the protective 
function attributed to the SEI, its formation entails a capacity loss as a consequence of the irreversible consumption of Lithium ions. The process is more pronounced during the first few cycles [179]. Among the factors affecting the SEI layer's evolution, a strong dependence on temperature and state of charge has been proved to exist for its morphology, composition and Lithium ionic conductivity [7, 37]. Continuum theory for the growth of an SEI layer have been recently proposed by many authors [128, 180, 181, 182, 183, 184, $77,185,186,187,188,189,190,191]$.

\subsection{The modeling of micro-mechanical effects.}

Since mechanical effects are among the most important mechanisms of capacity fade and impedance growth in Lithium-ion batteries [34], the stress generation during Lithium diffusion in active materials has been widely investigated. The intercalation process is generally modeled as a multicomponent system [192, 193], which comprises Lithium diffusing in a hosting material lattice. Atomic diffusion in solids is much slower than deformation [194], therefore mechanical equilibrium is postulated at all instants.

High capacity electrode materials, such as Silicon and Tin, manifest huge expansions when lithiated. Storage particles develop non-uniform Lithium concentration profiles, which cause differential strains and originate a stress field within the particle (referred to as chemical stresses [143] or diffusion-induced stresses [133]). Many efforts have been made in the last decade in order to understand and model the stress evolution in active particles [195]. Modeling involves multi-physics description of the processes as they entails coupling among mechanics, transport, and thermo-electro-chemical kinetics. Lithiation influences material parameters [107] and may lead to phase segregation, too, which causes lattice mismatch within the particle.

It has been observed that both anodic and cathodic materials may fracture during charging/discharging operations. Some electrode materials, such as Lithium-alloys, undergo decrepitation, i.e. fracturing in many small parts, caused by large deformations during lithiation [196]. Cracks have been observed even in active materials that undergo small deformations when lithiated, as for $\mathrm{LiCoO}_{2}, \mathrm{LiMn}_{2} \mathrm{O}_{4}$, and $\mathrm{LiFePO}_{4}[197]$

To predict the onset of fracture in electrode particles, many authors [198, 199, 196, 200, 201, 202, 197, 203] resorted to linear-elastic fracture mechanics [204, 205, 206, 207]. A pre-existing flaws population in the particles was assumed and Griffith's criterion used to investigate the effects of charging rate and fracture toughness on the failure of particles of different sizes. Assumptions have been often taken upon the most dangerous flaw orientation, which might be incorrect [208]. Alternatively, some authors adopted cohesive models for crack nucleation inside the electrode [209, 210, 127] or phase-field methods [211, 212, 213, 214, $215,216,217,218,219]$.

Other studies lead to design criteria in order to avoid particle fracturing based on the stresses generated inside the electrode. For example tensile stresses [135, 220] or von Mises equivalent stresses [143] have been taken as measures of the distance to the onset of fracture.

In what follows, homogeneous material formulations and phase segregation models will be separately described.

\subsubsection{Lithiation models in homogeneous particles.}

It is common in literature to idealize the geometry: particles are either modeled as spheres [133, 134, 220, 135, 136, 139, 141, 221], cylinders [210, 222], or thin films [209, 30]. Chemo-mechanical features are also simplified: materials are often taken to be isotropic with properties (Young's modulus, Poisson's ratio, diffusivities) insensitive to Lithium content [133, 134, 137, 139, 141, 142, 143].

Since Lithium transport is severely influenced by the deformation of the hosting material and vice-versa, diffusion and mechanics are entangled processes. Accordingly, the thermodynamic forces that drive Lithium diffusion shall be mathematically coupled to mechanical deformation. The coupling is usually described in analogy with thermo-mechanics, i.e. the variation of solute atoms concentration in the hosting material causes a pure volumetric deformation: the partial molar volume plays the same role of the thermal expansion coefficient [223, 224, 225, 226].

Whereas most early models neglected the role of mechanics, a few recent ones couple the mass transport to the mechanics in a one-directional way. In those models, the driving force for the diffusion is solely 


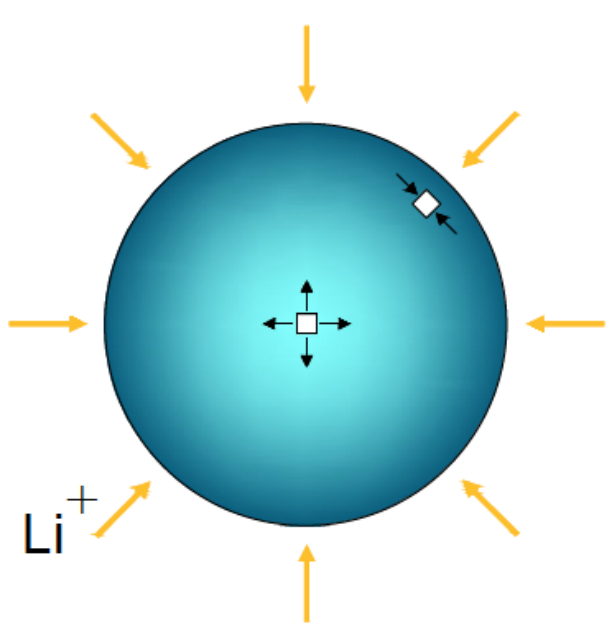

a) Lithiation

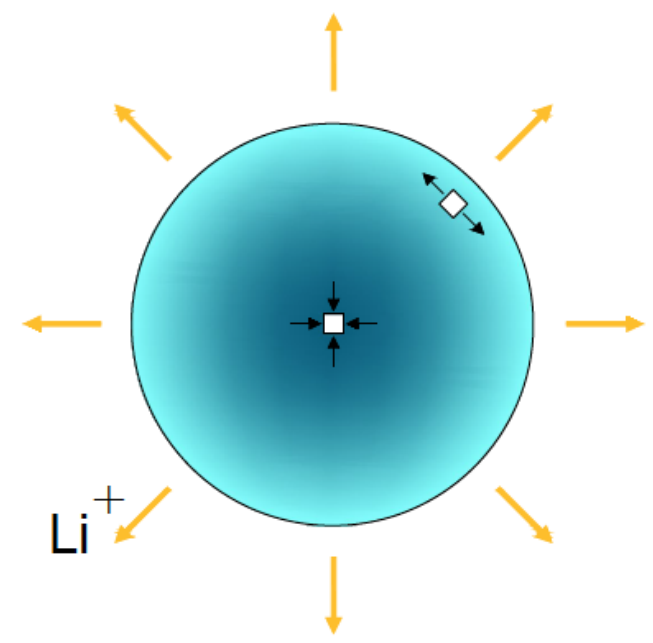

b) Delithiation

Figure 5: Schematic of particle expansion during Lithium insertion (e.g., during charge) and contraction during Lithium extraction (e.g., during discharge). Arrows pointing toward each other indicate regions of compression in the particle, while arrows pointing away from each other indicate regions of tension. Concentric circles mark host-lattice tags, which are initially evenly spaced [135].

the concentration gradient, therefore the Lithium concentration can be evaluated by solving the transport problem, while the chemical stresses can be computed afterwards. For linear-elastic material with parameters independent upon Lithium concentration, analytical solutions are attainable for simple geometries. This approach was taken in $[133,134]$, to investigate the critical factors that influence the overall response of active materials. Diffusion induced stresses were computed in spherical active particles under either galvanostatic or potentiostatic control, as well as under more realistic boundary Butler-Volmer kinetics. It was shown that during lithiation the Lithium concentration decreases from the outer surface to the center, inducing the outer shell to swell and generating compressive hoop stresses near the particle surface and tensile stress into the inner core, in view of geometrical compatibility, as described in Figure 5. The opposite during delithiation. The stress magnitude was found to depend on the charge/discharge rate [141] and on the particle size.

The effects of surface mechanics in nano-sized spherical particles were studied in [194, 221], in order to design particle of longer cyclability. It resulted that the magnitude and distribution of stresses can be significantly affected by surface mechanics if the particle is in the nanometer range. Such an approach was applied to nanowire electrodes [137], too.

Two-way coupling was considered in more recent papers. The mass flux is modeled as dependent on both concentration and hydrostatic pressure gradients, as in the inspiring framework of Larche and Cahn [227, 228]. Some authors assumed that particles remain linear-elastic during the whole process [139, 229, 230, 142, 143]. This assumption is reasonable for electrodes with moderate swelling, as LiC, LMO. Zhang and co-workers simulated the charging/discharging process of $\mathrm{Li}_{x} \mathrm{Mn}_{2} \mathrm{O}_{4}$ for $0 \leq x \leq 1$ and captured the range in which phase transitions occur as a function of the state of charge. The stress generation in three-dimensional ellipsoidal particles with different aspect ratios was evaluated through numerical simulations [143]. The numerical outcomes suggest that larger aspect ratio and smaller particle size may reduce the intercalation induced stresses.

The influence of particle morphology has been simulated in [230] after digitalization of real particles. The maximum von Mises stress induced by the lithiation in a digitalized particle turned out to be one order of magnitude higher than the one predicted on idealized shapes.

Miehe and Dal [140] proposed a computational theory accounting for electro-chemo-mechanical inter- 
action of Silicon particles. Numerical simulations shown significant discrepancy between small and finite strains approaches, pointing out the importance of large strains theory in modeling materials with significant swelling.

Silicon thin-films and particles show a markedly inelastic behavior during charge/discharge. Plasticity seem to infer a specific failure mechanism, since for spherical particles the initial compressive hoop stress in the outer shell during intercalation may even reverse [136]. The Lithium transport problem was coupled to plasticity models at infinitesimal [203] as well as at finite strains [231, 30, 127, 128, 136, 222, 232, 233, 234, 144].

Bower and co-workers [30] developed a general model that accounts for finite strains and plastic flow in electrodes materials. A Silicon thin-film was studied and compared with experimental evidences [235]. Cui et al. [136] focused on the chemical potential in the framework of finite strains and plastic flow as well. They proposed a chemical potential that extends the formulation of Larché-Cahn. Numerical examples on spherical Silicon particles emphasized the role of plastic flow in the lithiation process.

\subsubsection{Models accounting for phase-segregation}

Multi-phase models aim at investigating the behavior of particles in which phases co-exist because of lithiation. They essentially differ for Lithium content and for the crystal structure, which is transformed after intercalation [236]. The thickness of the physical region that separates distinct phases, the so-called phase boundary, varies upon materials. For instance, crystalline Silicon reacts with Lithium forming an amorphous phase with atomically sharp reaction front - about $1 \mathrm{~nm}$ thick [33]. Experimental evidences for $\mathrm{LiFePO}_{4}$ show a wider phase boundary, of several nanometers [237, 238].

The widespread class of so-called sharp-interface models splits particles in two distinct regions and idealizes the phase boundary to a zero-thickness interface. The class of phase-field models, usually stemming from the Cahn-Hilliard theory [239], smears the phase boundary in a narrow region of finite thickness, avoiding localized discontinuities. Alternative models recover discontinuous concentration profiles without recourse to any of the methods above. A review of modeling methods for phase boundary can be found in Thornton et al. [240].

Sharp-interface models [241, 242, 243] shown the ability to reproduce the observed voltage plateau [107] in the discharge profile of iron-phospate [241] and Lithium cobalt oxide [243] electrodes. To account for the phase transition, electrodes were modeled with the so called shrinking-core particle approach[242], which idealizes active particles as spheres with two distinct phases occupying the outer shell and the inner core, respectively[241]. The interface location is one of the unknowns of the problem and evolves in the radial direction driven by the Lithium transport in the Li-rich phase and on the concentration gap at the interface. Mechanical effects are included in the shrinking-core approach [220, 138, 112]. Particles have been modeled as linear-elastic bodies with different chemo-mechanical properties between the two phases. The onset of chemical stresses was modeled with the analogy to thermal stresses as in the single-phase models, therefore the stress field affects the phase-boundary kinetics. These models are capable to predict the rise of tensile stresses in the core-phase and compressive hoop stress in the outer shell during lithiation. Differently from single-phase models, the hoop stress can be discontinuous at the phase-boundary. The interfacial stress discontinuity depends upon the interface location, equilibrium concentration, and the material properties of the two phases.

In the phase-field models the dynamics of phase-segregation is ruled by the free-energy of the system. Differently from the Fickian description of diffusion, the Helmholtz free energy includes an interface energy, related to the gradient of concentration. Phase-segregation is not imposed a priori via sharp interfaces but arises as a consequence of the thermodynamic evolution of the system[244].

Early works applied the conventional Cahn-Hilliard phase-field model to describe phase separation in $\mathrm{LiFePO}_{4}$ electrodes $[245,246,247]$. In particular Singh et. al [245] showed the ability of this theory to model the anisotropic ionic mobility in single crystals. The Cahn-Hilliard theory was extended in order to assess the conditions for phase-segregation in electrode particles. The total free energy was modeled accounting for additional terms, such as the strain and surface energies. In this way Tang et al. [248, 249] and Cogswell and Bazant [250] investigated the effect of particle size, mechanics, and applied overpotential in the phase transition pathways of storage particles. 
Anand and co-workers [251] developed a general thermodynamically consistent theory that couples the Cahn-Hilliard theory with large elasto-plastic deformation mechanics. Simulations show the phasetransformation of a three dimensional $\mathrm{LiFePO}_{4}$ spheroidal particle [252].

Basic computational aspects of phase-segregation modeling in electrodes have been recently dealt with in [253].

Stress evolution in two-phase electrodes was also modeled alternatively. A sharp-interface was reproduced either creating a series of step-like concentration profiles [254], by choosing a concentration dependent parameters [255], or making recourse to the concept of Lithium traps [256, 257]. Active particles, mostly idealized as spheres, were assumed to deform visco-plastically, to mimic the mechanics of lithiated Silicon. Huang et al. [254] showed that the initial compressive hoop stress in the lithiated shell could be reversed if the material undergoes plastic deformations. Yang et al. [255] adopted a concentration dependent diffusivity and imposed that Li diffusivity in the lithiated region is much larger than in the pristine core. This induces the concentration profile to assume a typical pattern of a two-phase system, with interface tracking controlled by diffusion. Drozdov [256, 257] assumes that Lithium within the particle is separated in mobile and alloyed. The latter is treated as a kinetic process, which ultimately permits to recover a sharp interface.

More recent models of lithiation in Silicon describe the phase-boundary kinetics [32, 258, 259] and the observed anisotropy in intercalation [260]. Crystalline Silicon becomes amorphous upon lithiation, and diffusion through the amorphous phase has been observed to be faster than the reaction at interface [259]. Therefore the phase-boundary motion, differently from the shrinking-core models, shall not be controlled by Lithium diffusion. Zhao et al. [259] formulated the driving force of the reaction between pristine Silicon and Lithium at the interface including the effects of the stress field. By simple benchmarks they showed that the interface motion could be inhibited by the stress field generated in the particle. With a more general approach Cui and co-workers [258] developed a two-phase model for Silicon particles accounting for diffusion in both phases, stress generation and phase-boundary kinetics. The kinetics of reaction at interface and diffusion in both phases are concurrent processes. Their numerical results showed the impact of different ratios between rates of interface kinetics and bulk diffusion in Lithium distribution and stress generation.

\section{Multiscale models}

Intercalation, swelling, and eventually the mechanical failure originate at a scale three order of magnitudes smaller than the battery cell scale, at which ion mobility is usually modeled. Since modeling a whole battery cell at the nano-scale is computationally unfeasible, nano-scale effects are incorporated into the microscale problem through homogenization approaches and constitutive models that are derived from multiscale approaches. They frame on a representative volume element (RVE), in which the relevant features of the microscopic morphology are accounted for. The proper selection of the RVE is a fundamental ingredient, as usual in the theory of composite materials. According to [261], an RVE may be defined in two different ways. It can be considered as the smallest microstructural volume for which the averages of properties represent with "sufficient accuracy" the mean macroscopic response. Accordingly, RVE's size is influenced by the material behavior of the microstructural components. A second definition requires the RVE to be statistically representative of the microstructure, that is to essentially include a sampling of all possible microstructural configurations occurring in the composite. This definition leads to significantly larger RVEs than in the former case, as the microstructural element must incorporate several kinds of material heterogeneities. Based on this definition, statistical methods have been presented to determine the size of the RVE and the number of inclusions to consider [262, 263, 264, 265].

Representative models of the battery microstructure were based on experimentally obtained statistical information. The three dimensional microstructure of a graphite porous electrode was reconstructed recurring to tomographic techniques[157]. The minimum RVE size was calculated as $43 \times 60 \times 60 \mu \mathrm{m}$ after evaluating several geometrical parameters (e.g. porosity, pore and particle size distribution) and extracting the relative standards deviations. An estimate of the characteristic dimensions of a cubic RVE for $\mathrm{LiCoO}_{2}$ electrodes was derived in [149], resulting in an edge of $\approx 30 \mu \mathrm{m}$. A similar approach [266] was employed for $\mathrm{LiFePO}_{4}$ electrodes, $5 \times 5 \times 15 \mu \mathrm{m}$. 
Ferguson and Bazant [267] made use of Wiener and Hashin-Shtrikman bounds to characterize the electrical conductivity and tortuosity of porous media, while an asymptotic multiscale expansion was pursued by Schmuck and Bazant [268].

Wieser et al. [155] made use of 3D imaging at different scales and homogenization techniques to estimate effective transport properties of ionic species within porous electrodes. The authors identified a micrometer porosity for the composite electrodes - made up by solid active material, additives and open pores filled by the liquid electrolyte - and a nanometer porosity within the additives - a mixture of carbon conductive particles and polymeric binder. A lithium metal/separator/graphite-based porous electrode half-cell was simulated showing that the impact of additives nanometer porosity on transport limitation is negligible.

Awarke et al. [145] attempted to quantify the impact of changes in the arrangement of solid particles within Li-ion battery electrode on the conductivity and tortuosity of a $\mathrm{LiFePO}_{4}$ based cathode. A Finite Element Method (FEM) was used to analyze RVEs made up by spherical particles with various arrangements representing the same porous agglomerate under different mechanical and electrical loading conditions. Volume averages have been used to compute effective macroscopic properties (elastic tensor, volume expansion coefficient and electrical conductivity) which have in turn been adopted in numerical analyses on a cathode sample.

Lee et al. [269] adapted the variational multi-scale principle to a Li-ion battery system, in order to improve the predictions of battery performance by including multiphysics phenomena among the particle aggregates in the electrode. The role of the microstructure was highlighted, in terms of particle shape, tortuosity, and material composition.

A continuum model for Li-ion battery accounting for electrochemical and mechanical effects at multiple scales was presented by Golmon and coworkers [270]. Transport processes and battery deformation have been modeled at the cell scale, where the mechanical interactions with surrounding layers was considered through tractions boundary conditions. A single spherical active particle was analyzed to account for the microscopic evolution of the system both in terms of intercalated Lithium (state of charge) and chemically induced local stresses. The macroscopic pore wall flux was related to the microscale following Newman's porous electrode theory, while the Mori-Tanaka effective-field theory was used to relate mechanical properties between scales. Both scales were idealized to one-dimensional problems as in pseudo-2D models. The electrochemical and mechanical performance of half-cell have been studied by varying the electrochemical properties, the cathode particle radius, the porosity of the cathode, the discharge current density and the mechanical boundary conditions. The model has been further applied to design optimization of a Li-foil/separator/porous electrode battery [271] and to a battery cell in which both electrodes were porous [272].

Volume averaging techniques that are applicable to the entire cell with arbitrary 3D electrode configuration have been recently proposed [273].

Gupta et al. [146] proposed an electrochemical model for porous electrodes that accounts for two different scales. At the microscopic scale an RVE made up by an electrolyte and a solid matrix domain was identified for the porous electrode. Governing equations for Lithium and electric charges were written over each domain moving from conservation equations typical of continuum models. The cell scale governing equations differ from the ones detailed in $[67,68]$ because the volume-averaged fluxes have been explicitly provided through the introduction of effective transport properties, such as the Bruggeman's equation [72]. This approach differs from pseudo-2D models because all the macroscopic variables have been derived from microscopic volume averaging.

In recent contributions, $[67,68]$ a computational homogenization $(\mathrm{CH})$ technique was tailored to Li-ion batteries by using a multiscale scheme with a complex multi-particle RVE idealizing active particles in the composite electrodes as network solids following Larche and Cahn [227], with the lattice material assumed as insoluble in the electrolyte. The $\mathrm{CH}$ technique is based on the solution of nested boundary value problems, one for each scale. A complete set of equations and boundary conditions governing the stress, electric, chemical, and electrochemical potentials was derived [67] for the whole battery cell for both scales following non-equilibrium thermodynamics of porous electrodes [267, 64]. Use of the Butler-Volmer equation was made to describe the intercalation kinetics accounting for the flux of Lithium between the particles and the electrolyte, which at the macro scale was modeled as a bulk supply. At such a scale, transport and stress evolution were modeled via volume averaged conservation equations.

In the rigorous mathematical formulation of multi scale modeling - see $[274,275]$ - the micro to macro scale transition requires conservation of power expenditure between both scales, thus assuring that energy is 
neither artificially generated nor artificially dissipated across the scales. If electro-neutrality is used in place of Maxwell's equations, recovering the energy description of the electromagnetic interactions is not possible. Therefore, electroneutrality assumption (2) cannot be used in multi scale approaches [68]. In the approach proposed in $[67,68]$, rather then imposing the electroneutrality condition as an equation to be fulfilled, the impact that it has on the fundamental balance laws was investigated. Lorentz forces were thus neglected, whereas it was shown that electroneutrality has no influence on Maxwell's law.

A rigorous analysis of general principles of non-equilibrium thermodynamics $[24,276]$ has been performed in $[55,56]$. The electrochemical potential was defined moving from the rate at which power is expended on a material region, in terms of mechanical contribution as well as of the power due to mass transport and to electromagnetic interactions. All processes were taken to be isothermal. The entropy imbalance with the Coleman-Noll procedure provide thermodynamic restrictions, satisfied by the usual Fickian description of diffusion and migration in terms of electrochemical potential, defined as in [22, 23]. Infinitely dilute solutions as well as solutions close to saturation have been numerically simulated.

A mixture theory has been presented in [131] for a liquid solvent containing (completely) dissociated ions. Electro-mechanical interaction has been explicitly taken into account by introducing the Lorentz contribution within the balance of forces and a chemical potential dependence on pressure. The effect of solvation was also considered in the definition of entropy of mixing. A fully coupled model for charge, species and thermal transport in Li-ion batteries has been developed by Latz and coworkers [170]. The formulation, based on general principles of non-equilibrium thermodynamics, makes use of charge neutrality assumption for both electrolyte and active particles. Electrical, chemical and thermal interactions between electrolyte and active particle were taken into account by proper interface conditions.

\section{Conclusions}

The relevance and timeliness of modeling and simulations in the field of energy storage materials [277] is made evident by the intense flow of scientific publications. A review of this abundant literature may reveal thus useful, although keen to become soon obsolete. An effort was provided in this note, moving from the inherent multi-scale nature of $\mathrm{Li}$-ion batteries.

Continuum thermo-chemo-electro-mechanical models have been discussed at different scales and multiscale approaches have been analyzed as well. This study illustrated the progresses made since the pioneering publications, and made clear that modeling is becoming more and more accurate and predictive and, with the availability of high performance computing, it can integrate experimental campaigns in discovering new materials and developing new architectures.

The non-equilibrium thermodynamics, coupled with rigorous scale transitions, is the appropriate theoretical background for multi-scale and multi-physics modeling. The future scientific endeavors will stem from this fundamental framework. They are expected to finalize the three-dimensional multi-scale approaches currently in progress into high-performance computing scalable codes, in order to investigate major concerns in current batteries technology as the behavior at high C-rates and voltages, which may lead to thermal runaway fueled by side reactions. A clear understanding of these phenomena may provide significant progresses in batteries safety, particularly under abuse or extreme conditions.

Several aging phenomena in batteries require further scientific investigations. These study may address some fundamental problems of electrode chemo-mechanical instabilities that have so far limited the power, energy, and durability of advanced batteries. The modeling of Lithium deposition and dendritic growth, for instance, is particularly relevant for the safe use of Lithium metal anodes yet lags behind the experimental evidences, in spite of recent investigations[91]. Co-designed experiments and simulations may pave the way to a deeper understanding of these limiting phenomena.

The predictive ability of modeling and simulations relies on the realistic reconstruction of three-dimensional porous electrode. Evident progresses have been made in this recent years, particularly in the field of X-ray tomography. The required accuracy of the electrode reconstruction is strictly related to the targeted processes, so that the classical statistical paradigms of homogenization may not always be applicable successfully.

This review did not focus on atomistic simulations. A major challenge of the future investigations will concern the incorporation of quantum mechanics and molecular dynamics into coarser scales formulations. 
As pointed out also in $[278,8]$, it will become more and more important to develop multi-scale models that account for a realistic chemical environment by means of coupling discrete and continuum approaches. This seems to be particularly relevant in the modeling the interface phenomena, that take place in atomistic-size narrow layers.

Modeling unavoidably requires identification of material parameters, most of which can hardly be measured experimentally[279] especially in operating conditions [280]. Severe procedures of calibration must be put in place in order to ensure that modeling indeed achieves predictive science capability. In this regard, computational modeling and simulations may take advantage of the most recent achievements in the fields of uncertainty quantification and sensitivity analysis.

\section{Acknowledgements}

We express our deep gratitude to Prof. B. Scrosati, who inspired us and proposed to write this review. He also revised part of the manuscript. FIB-SEM analyses in figure 4 were made in cooperation with J.Pauls, A. Mukasyan, J.Schaefer, and K. Matouš at the University of Notre Dame, USA.

\section{References}

[1] B. Scrosati and J. Garche. Lithium batteries: status, prospects and future. J POWER SOURCES, 195:2419-2430, 2010.

[2] J.M. Tarascon and M. Armand. Issues and challenges facing rechargeable Lithium batteries. NATURE, 414:359-367, 2001.

[3] A. Danzer, V. Liebau, and F. Maglia. Aging of Lithium-ion batteries for electric vehicles. In B. Scrosati, J. Garche, and W. Tillmetz, editors, Advances in battery technologies for electric vehicles, pages 359387. Woodhead Publishing, 2015.

[4] A. Salvadori and D. Grazioli. Computer simulation for battery design and lifetime prediction. In B. Scrosati, J. Garche, and W. Tillmetz, editors, Advances in battery technologies for electric vehicles, pages 417-442. Woodhead Publishing, 2015.

[5] L. Lu, X. Han, J. Li, J. Hua, and M. Ouyang. A review on the key issues for Lithium-ion battery management in electric vehicles. J POWER SOURCES, 226(0):272 - 288, 2013.

[6] J. Zhang and J. Lee. A review on prognostics and health monitoring of Li-ion battery. J POWER SOURCES, 196(15):6007 - 6014, 2011.

[7] A. Barre, B. Deguilhem, S. Grolleau, M. Gerard, F. Suard, and D. Riu. A review on Lithium-ion battery ageing mechanisms and estimations. J POWER SOURCES, 241:680-689, 2013.

[8] A.A. Franco. Multiscale modelling and numerical simulation of rechargeable Lithium ion batteries: concepts, methods and challenges. RSC ADVANCES, 3(13027), 2013.

[9] M. Landstorfer and T. Jacob. Mathematical modeling of intercalation batteries at the cell level and beyond. CHEM SOC REV, 42:3234-3252, 2013.

[10] D. Doerffel and S.A. Sharkh. A critical review of using the Peukert equation for determining the remaining capacity of lead-acid and Lithium-ion batteries. J POWER SOURCES, 155(2):395-400, 2006.

[11] W Peukert. Über die abhängigkeit der kapazität von der entladestromstärke bei bleiakkumulatoren. Elektrotech Z, 1897.

[12] M. Chen and G. Rincon-Mora. Accurate electrical battery model capable of predicting runtime and IV performance. IEEE T ENERGY CONVER, 21(2):504-511, 2006. 
[13] R. L. Fares and M.E. Webber. Combining a dynamic battery model with high-resolution smart grid data to assess microgrid islanding lifetime. APPL ENERG, 137:482-489, 2015.

[14] P. Rong, S. Member, and M. Pedram. An analytical model for predicting the remaining battery capacity of Lithium-ion batteries. IEEE T VLSI SYST, 14(5):441-451, 2006.

[15] M.T. von Srbik, M. Marinescu, R.F. Martinez-Botas, and G.J. Offer. A physically meaningful equivalent circuit network model of a Lithium-ion battery accounting for local electrochemical and thermal behaviour, variable double layer capacitance and degradation. J POWER SOURCES, 325:171 - 184, 2016.

[16] W.D. Widanage, A. Barai, G.H. Chouchelamane, K. Uddin, A. McGordon, J. Marco, and P. Jennings. Design and use of multisine signals for Li-ion battery equivalent circuit modelling. Part II: Model estimation. J POWER SOURCES, 324:61 - 69, 2016.

[17] H. He, R. Xiong, and J. Fan. Evaluation of Lithium-ion battery equivalent circuit models for state of charge estimation by an experimental approach. Energies, 4:582-598, 2011.

[18] L. Gao, S. Liu, and R.A. Dougal. Dynamic Lithium-ion battery model for system simulation. IEEE T COMPON PACK T, 25(3):495-505, 2002.

[19] S. Buller and M. Thele. Impedance-based simulation models of supercapacitors and Li-ion batteries for power electronic applications. IEEE T IND APPL, 41(3):742-747, 2005.

[20] S. Gold. A PSPICE macromodel for Lithium-ion batteries. In The $12^{\text {th }}$ annual battery conference on applications and advances., pages 215-222, 1997.

[21] A. Fotouhi, D.J. Auger, K. Propp, S. Longo, and M. Wild. A review on electric vehicle battery modelling: From Lithium-ion toward Lithium-sulphur. RENEW SUST ENERG REV, 56:1008 - 1021, 2016.

[22] S.R. De Groot and P. Mazur. Non-Equilibrium Thermodynamics. Dover, 1984.

[23] R. DeHoff. Thermodynamic in material science. CRC Press - Taylor and Francis, 2006.

[24] M.E. Gurtin, E. Fried, and L. Anand. The Mechanics and Thermodynamics of Continua. Cambridge University Press, 2010.

[25] S. Shell. Thermodynamics and statistical mechanics: an integrated approach. Cambridge University Press, 2015.

[26] E.B. Tadmor, R.E. Miller, and R.S. Elliott. Continuum Mechanics and Thermodynamics: From Fundamental Concepts to Governing Equations. Cambridge University Press, 2011.

[27] P. Tagade, K.S. Hariharan, S. Basu, M.K.S. Verma, S.M. Kolake, T. Song, D. Oh, T. Yeo, and S.K. Doo. Bayesian calibration for electrochemical thermal model of Lithium-ion cells. J POWER SOURCES, 320:296 - 309, 2016.

[28] C. Edouard, M. Petit, C. Forgez, J. Bernard, and R. Revel. Parameter sensitivity analysis of a simplified electrochemical and thermal model for Li-ion batteries aging. J POWER SOURCES, 325:482 - 494, 2016.

[29] A.J Bard and L.R. Faulkner. Electrochemical Methods: Fundamentals and Applications. Wiley, 2nd edition, 2000.

[30] A.F. Bower, P.M. Guduru, and V.A. Sethuraman. A finite strain model of stress, diffusion, plastic flow and electrochemical reactions in a Lithium-ion half-cell. J MECH PHYS SOLIDS, 59:804-828, 2011.

[31] A. Aurbach. The role of surface films on electrodes in Li-ion batteries. In W. van Schalkwijk and B. Scrosati, editors, Advances in Lithium-ion batteries. Kluwer Academic, New York, 2002. 
[32] A.F. Bower, P.R. Guduru, and E. Chason. Analytical solutions for composition and stress in spherical elastic-plastic Lithium-ion electrode particles containing a propagating phase boundary. INT J SOLIDS STRUCT, 69-70:328-342, 2015.

[33] M.J. Chon, V.A. Sethuraman, A. McCormick, V. Srinivasan, and P.R. Guduru. Real-time measurement of stress and damage evolution during initial lithiation of crystalline silicon. PHYS REV LETT, 107:045503, 2011.

[34] P. Arora, R.E. White, and M. Doyle. Capacity fade mechanisms and side reactions in Lithium-ion batteries. J ELECTROCHEM SOC, 145(10):3647-3667, 1998.

[35] R. Marom, S.F. Amalraj, N. Leifer, D. Jacob, and D. Aurbach. A review of advanced and practical Lithium battery materials. J MATER CHEM, 21:9938-9954, 2011.

[36] G. Sarre, P. Blanchard, and M. Broussely. Aging of Lithium-ion batteries. J POWER SOURCES, 127:65-71, 2004.

[37] J. Vetter, P. Novak, M.R. Wagner, C. Veit, K.C. Moeller, J.O. Besenhard, M. Winter, M. WohlfahrtMehrens, C. Vogler, and A. Hammouche. Ageing mechanisms in Lithium-ion batteries. J POWER SOURCES, 147(1-2):269-281, 2005.

[38] M. Wohlfart-Mehrens, C. Vogler, and J. Garche. Aging mechanism of Lithium cathode materials. J POWER SOURCES, 127:58-64, 2004.

[39] J.G. Swallow, W.H. Woodford, F.P. McGrogan, N. Ferralis, Y.M. Chiang, and K.J. Van Vliet. Effect of electrochemical charging on elastoplastic properties and fracture toughness of $\mathrm{Li}_{x} \mathrm{CoO}_{2}$. $\underline{\mathrm{J}}$ ELECTROCHEM SOC, 161(11):F3084-F3090, 2014.

[40] J.G. Swallow, W.H. Woodford, Y. Chen, Q. Lu, J.J. Kim, D. Chen, Y.M. Chiang, W.C. Carter, B. Yildiz, H.L. Tuller, and K.J. Van Vliet. Chemomechanics of ionically conductive ceramics for electrical energy conversion and storage. J ELECTROCERAM, 32:3-27, 2014.

[41] W.I.F. David, M.M. Thackeray, L.A. De Picciotto, and J.B. Goodenough. Structure refinement of the spinel-related phases $\mathrm{Li}_{2} \mathrm{Mn}_{2} \mathrm{O}_{4}$ and $\mathrm{Li}_{0.2} \mathrm{Mn}_{2} \mathrm{O}_{4}$. J SOLID STATE CHEM, 67(2):316-323, 1987.

[42] T. Ohzuku, Y. Iwakoshi, and K. Sawai. Formation of Lithium-graphite intercalation compounds in nonaqueous electrolytes and their application as a negative electrode for a Lithium ion (shuttlecock) cell. J ELECTROCHEM SOC, 140(9):2490-2498, 1993.

[43] T. Ohzuku, N. Matoba, and K. Sawai. Direct evidence on anomalous expansion of graphite-negative electrodes on first charge by dilatometry. J POWER SOURCES, 97-98:73-77, 2001. Proceedings of the 10th International Meeting on Lithium Batteries.

[44] H. Wang, Y.I. Jang, B. Huang, D.R. Sadoway, and Y.M. Chiang. TEM study of electrochemical cycling-induced damage and disorder in $\mathrm{LiCoO}_{2}$ cathodes for rechargeable Lithium batteries. $\underline{\mathrm{J}}$ ELECTROCHEM SOC, 146(2):473-480, 1999.

[45] M.C. Tucker, J.A. Reimer, and E.J. Cairns. A study of capacity fade in metal-substituted Lithium manganese oxide spinels. J ELECTROCHEM SOC, 149(5):A574-A585, 2002.

[46] M.R. Lim, W.I. Cho, and K.B. Kim. Preparation and characterization of gold-codeposited $\mathrm{LiMn}_{2} \mathrm{O}_{4}$ electrodes. J POWER SOURCES, 92(1-2):168-176, 2001.

[47] D. Wang, X. Wu, Z. Wang, and L. Chen. Cracking causing cyclic instability of $\mathrm{LiFePO}_{4}$ cathode material. J POWER SOURCES, 140(1):125-128, 2005.

[48] H. Gabrisch, J. Wilcox, and M. M. Doeff. TEM study of fracturing in spherical and plate-like $\mathrm{LiFePO}_{4}$ particles. ELECTROCHEM SOLID ST, 11(3):A25-A29, 2008.

[49] R. Kostecki and F. McLarnon. Microprobe study of the effect of Li intercalation on the structure of graphite. J POWER SOURCES, 119-121:550-554, 2003. Selected papers presented at the 11th International Meeting on Lithium Batteries. 
[50] L.J. Hardwick, M. Marcinek, L. Beer, J.B. Kerr, and R. Kostecki. An investigation of the effect of graphite degradation on irreversible capacity in Lithium-ion cells. J ELECTROCHEM SOC, 155(6):A442-A447, 2008.

[51] E. Markervich, G. Salitra, M.D. Levi, and D. Aurbach. Capacity fading of lithiated graphite electrodes studied by a combination of electroanalytical methods, raman spectroscopy and SEM. J POWER SOURCES, 146(1-2):146-150, 2005. Selected papers presented at the 12th International Meeting on Lithium Batteries12th International Meeting on Lithium Batteries.

[52] L. Y. Beaulieu, K. W. Eberman, R. L. Turner, L. J. Krause, and J. R. Dahn. Colossal reversible volume changes in Lithium alloys. ELECTROCHEM SOLID ST, 4(9):A137-A140, 2001.

[53] M. Tang, P. Albertus, and J. Newman. Two-dimensional modeling of Lithium deposition during cell charging. J ELECTROCHEM SOC, 156:A390-A399, 2009.

[54] D. P. Finegan, M. Scheel, J. B. Robinson, B. Tjaden, I. Hunt, T. J. Mason, J. Millichamp, M. Di Michiel, G. J. Offer, G. Hinds, D. J. L. Brett, and P. R. Shearing. In-operando high-speed tomography of Lithium-ion batteries during thermal runaway. NAT COMMUN, 6, 042015.

[55] A. Salvadori, D. Grazioli, M.G.D. Geers, D. Danilov, and P.H.L Notten. A novel approach in modeling ionic transport in the electrolyte of (Li-ion) batteries. J POWER SOURCES, 293:892-911, 2015.

[56] A. Salvadori, D. Grazioli, M. Magri, M.G.D. Geers, D. Danilov, and P.H.L. Notten. On the role of saturation in modeling ionic transport in the electrolyte of (Li-ion) batteries. J POWER SOURCES, 294:696-710, 2015.

[57] S.S. Zhang. A review on the separators of liquid electrolyte Li-ion batteries. J POWER SOURCES, 164:351-364, 2007.

[58] W. Wu, X. Xiao, X. Huang, and S. Yan. A multiphysics model for the in situ stress analysis of the separator in a Lithium-ion battery cell. COMP MATER SCI, 83:127-136, 2014.

[59] L.R. Valoen and J.N Reimers. Transport properties of $\mathrm{LiPF}_{6}$-based Li-ion battery electrolytes. $\underline{\mathrm{J}}$ ELECTROCHEM SOC, 152(5):A882-A891, 2005.

[60] G. Bauer, V. Gravemeier, and W.A Wall. A 3D finite element approach for the coupled numerical simulation of electrochemical systems and fluid flow. INT J NUMER METH ENG, 86:1339-1359, 2011.

[61] G. Bauer, V. Gravemeier, and W.A Wall. A stabilized finite element method for the numerical simulation of multi-ion transport in electrochemical systems. COMPUT METHOD APPL M, 223-224:199$210,2012$.

[62] A. Latz and J. Zausch. Multiscale modeling of Li-ion batteries: thermal aspects. BEILSTEIN J NANOTECHNOL, 6:987-1007, 2015.

[63] D. Danilov and P.H.L. Notten. Mathematical modeling of ionic transport in the electrolyte of Li-ion batteries. ELECTROCHIM ACTA, 53:5569-5578, 2008.

[64] J. Newman and K.E. Thomas-Alyea. Electrochemical systems. John Wiley and Sons B.V., 2004.

[65] M.Z. Bazant, K.T. Chu, and B.J. Bayly. Current-voltage relations for elecrochemical thin films. SIAM Journal of Applied Mathematics, 65:1463-1484, 2005.

[66] E.J.F. Dickinson, J.G. Limon-Petersen, and R.G. Compton. The electroneutrality approximation in electrochemistry. J SOLID STATE ELECTR, 15:1335-1345, 2011.

[67] A. Salvadori, E. Bosco, and D. Grazioli. A computational homogenization approach for Li-ion battery cells. Part 1 - Formulation. J MECH PHYS SOLIDS, 65:114-137, 2014.

[68] A. Salvadori, D. Grazioli, and M.G.D. Geers. Governing equations for a two-scale analysis of Li-ion battery cells. INT J SOLIDS STRUCT, 59:90-109, 2015. 
[69] D. Djian, F. Alloin, S. Martinet, H. Lignier, and J.Y. Sanchez. Lithium-ion batteries with high charge rate capacity: Influence of the porous separator. J POWER SOURCES, 172:416-421, 2007.

[70] X. Huang. Separator technologies for Lithium-ion batteries. J SOLID STATE ELECTR, 15:649-662, 2011.

[71] K.M.E Abraham. Directions in secondary Lithium battery research and development. ELECTROCHIM ACTA, 38:1233-1248, 1993.

[72] D.A.G. Bruggeman. Berechnung verschiedener physikallischer konstanten von heterogenen substanzen. ANN PHYS-LEIPZIG, 24:636-664, 1935.

[73] R.B. MacMullin and G.A. Muccini. Characteristics of porous beds and structures. AICHE J, 2:393-403, 1956.

[74] K.K. Patel, J.M. Paulsen, and J. Desilvestro. Numerical simulation of porous networks in relation to battery electrodes and separators. J POWER SOURCES, 122:144-152, 2003.

[75] I.V. Thorat, D.E. Stephenson, N.A. Zacharias, K. Zaghib, J.N. Harb, and D.R. Wheeler. Quantifying tortuosity in porous Li-ion battery materials. J POWER SOURCES, 188:592-600, 2009.

[76] X. Xiao, W. Wu, and X. Huang. A multi-scale approach for the stress analysis of polymeric separators in a Li-ion battery. J POWER SOURCES, 195:7649-7660, 2010.

[77] M.B. Pinson and M.Z. Bazant. Theory of SEI formation in rechargeable batteries: Capacity fade, accelerated aging and lifetime prediction. J ELECTROCHEM SOC, 160(2):A243-A250, 2013.

[78] J.W. Long, B. Dunn, D.R. Rolison, and H.S. White. Three-dimensional battery architectures. CHEM REV, 104:4463-4492, 2004.

[79] J.G. Kim, B. Son, S. Mukherjee, N. Schuppert, A. Bates, O. Kwon, M.J. Choi, H.Y. Chung, and S. Park. A review of lithium and non-lithium based solid state batteries. J POWER SOURCES, 282:299 - 322, 2015.

[80] A.L. Robinson. Solid-state batteries enter EV fray. MRS BULL, 39:1046-1047, 2014.

[81] B. Scrosati and C.A. Vincent. Polymer electrolytes: the key to Lithium polymer batteries. MRS BULL, March:28-30, 2000.

[82] M. Park, X. Zhang, M. Chung, G.B. Less, and A.M. Sastry. A review of conduction phenomena in Li-ion batteries. J POWER SOURCES, 195(24):7904-7929, 2010.

[83] S.D. Fabre, D. Guy-Bouyssou, P. Bouillon, F. Le Cras, and C. Delacourta. Charge/discharge simulation of an all-solid-state thin-film battery using a one-dimensional model. J ELECTROCHEM SOC, 159(2):A104-A115, 2012.

[84] V. Thangadurai, S. Narayanan, and D. Pinzaru. Garnet-type solid-state fast li ion conductors for li batteries: critical review. CHEM SOC REV, 43:4714-4727, 2014.

[85] N.J. Dudney and B.J. Neudecker. Solid state thin-film lithium battery systems. CURR OPIN SOLID ST M, 4:479-482, 1999.

[86] D. Danilov, R.A.H. Niessen, and P.H.L. Notten. Modeling all-solid-state Li-ion batteries. J ELECTROCHEM SOC, 158(3):A215-A222, 2011.

[87] Y. Wang, B. Liu, Q. Li, S. Cartmell, S. Ferrara, Z.D. Deng, and J. Xiao. Lithium and lithium ion batteries for applications in microelectronic devices: A review. J POWER SOURCES, 286:330 - 345, 2015 .

[88] Watanabe and Ogata. Ionic conductivity of polymer electrolytes and future applications. BRIT POLYM J, 20(3):181-192, 1988. 
[89] D.T. Hallinan Jr and N.P. Balsara. Polymer electrolytes. ANNU REV MATER RES, 43:503-525, 2013.

[90] P. Johansson. Computational modelling of polymer electrolytes: What do 30 years of research efforts provide us today? ELECTROCHIM ACTA, 175:42-46, 2015.

[91] P.P. Natsiavas, K. Weinberg, D. Rosato, and M. Ortiz. Effect of prestress on the stability of electrodeelectrolyte interfaces during charging in Lithium batteries. J MECH PHYS SOLIDS, 95:92 - 111, 2016.

[92] Giovanna Bucci, Yet-Ming Chiang, and W. Craig Carter. Formulation of the coupled electrochemicalmechanical boundary-value problem, with applications to transport of multiple charged species. ACTA MATER, 104:33 - 51, 2016.

[93] K.E. Thomas, J. Newman, and R.M. Darling. Matematical modeling of Lithium batteries. In W. van Schalkwijk and B. Scrosati, editors, Advances in Lithium-ion batteries. Kluwer Academic, New York, 2002 .

[94] J.S. Newman and C.W. Tobias. Theoretical analysis of current distribution in porous electrodes. J ELECTROCHEM SOC, 109(12):1183-1191, 1962.

[95] J. Newman and W.M. Tiedemann. Porous-electrode theory with battery applications. AICHE J, 21(1):25-41, 1975.

[96] M. Doyle and J. Newman. The use of mathematical modeling in the design of Lithium/polymer battery systems. ELECTROCHIM ACTA, 40(13):2191-2196, 1995.

[97] M. Doyle, T.F. Fuller, and J. Newman. Modeling of galvanostatic charge and discharge of the Lithium/polymer/insertion cell. J ELECTROCHEM SOC, 140:1526-1533, 1993.

[98] T.F. Fuller, M. Doyle, and J. Newman. Simulation and optimization of the dual Lithium ion insertion cell. J ELECTROCHEM SOC, 141(1):1-10, 1994.

[99] R.E. Garcia, Y.M. Chiang, W.C. Carter, P. Limthongkul, and C.M. Bishop. Microstructural modeling and design of rechargeable Lithium-ion batteries. J ELECTROCHEM SOC, 152:255-263, 2005.

[100] K. West, T. Jacobsen, and S. Atlung. Modeling of porous insertion electrodes with liquid electrolyte. J ELECTROCHEM SOC, 129(7):1480-1485, 1982.

[101] J. Christensen. Modeling diffusion-induced stress in Li-ion cells with porous electrodes. $\underline{J}$ ELECTROCHEM SOC, 157:366-380, 2010.

[102] V. Ramadesigan, P.W.C. Northrop, S. De, S. Santhanagopalan, R.D. Braatz, and V.R. Subramanian. Modeling and simulation of Lithium-ion batteries from a systems engineering perspective. J ELECTROCHEM SOC, 159(3):R31-R45, 2012.

[103] S. Atlung, K. West, and T. Jacobsen. Dynamic aspects of solid solution cathodes for electrochemical power sources. J ELECTROCHEM SOC, 126(8):1311-1321, 1979.

[104] M. Doyle, J. Newman, A. S. Gozdz, C. N. Schmutz, and J.M. Tarascon. Comparison of modeling predictions with experimental data from plastic Lithium ion cells. J ELECTROCHEM SOC, 143(6):1890-1903, 1996.

[105] P. Arora, M. Doyle, A.S. Gozdz, R.E. White, and J.Newman. Comparison between computer simulations and experimental data for high-rate discharges of plastic Lithium-ion batteries. J POWER SOURCES, 88(2):219 - 231, 2000.

[106] J. Smekens, J. Paulsen, W. Yang, N. Omar, J. Deconinck, A. Hubin, and J. Van Mierlo. A modified multiphysics model for Lithium-ion batteries with a $\mathrm{Li}_{x} \mathrm{Ni}_{1 / 3} \mathrm{Mn}_{1 / 3} \mathrm{Co}_{1 / 3} \mathrm{O}_{2}$ electrode. ELECTROCHIM ACTA, 174:615-624, 2015. 
[107] A. Mukhopadhyay and B.V. Sheldon. Deformation and stress in electrode materials for Li-ion batteries. PROG MATER SCI, 63:58-116, 2014.

[108] T.F. Fuller, M. Doyle, and J. Newman. Relaxation phenomena in Lithium-ion-insertion cells. J ELECTROCHEM SOC, 141(4):982-990, 1994.

[109] M. Doyle and Y. Fuentes. Computer simulations of a Lithium-ion polymer battery and implications for higher capacity next-generation battery designs. J ELECTROCHEM SOC, 150(6):A706-A713, 2003.

[110] S. Pramanik and S. Anwar. Electrochemical model based charge optimization for Lithium-ion batteries. J POWER SOURCES, 313:164 - 177, 2016.

[111] K.E. Thomas and J. Newman. Thermal modeling of porous insertion electrodes. J ELECTROCHEM SOC, 150(2):A176-A192, 2003.

[112] S. Renganathan, G. Sikha, S. Santhanagopalan, and R. E. White. Theoretical analysis of stresses in a Lithium ion cell. J ELECTROCHEM SOC, 157:155-163, 2010.

[113] H. Arunachalam, S. Onori, and I. Battiato. On veracity of macroscopic Lithium-ion battery models. J ELECTROCHEM SOC, 10:A1940-A1951, 2015.

[114] R. E. Garcia and Y.M. Chiang. Spatially resolved modeling of microstructurally complex battery architectures. J ELECTROCHEM SOC, 154(9):A856-A864, 2007.

[115] C.W. Wang and A.M. Sastry. Mesoscale modeling of a Li-ion polymer cell. J ELECTROCHEM SOC, 154:A1035-A1047, 2007.

[116] R.T. Purkayastha and R.M. McMeeking. An integrated 2-D model of a Lithium ion battery: the effect of material parameters and morphology on storage particle stress. COMPUT MECH, 50:209-227, 2012 .

[117] O. Stern. Zur theorie der elektrolytischen doppelschicht. Z Elektrochem, 30(508-516), 1924.

[118] D.L. Chapman. A contribution to the theory of electrocapillarity. PHILOS MAG, 25:475-481, 1913.

[119] M. Gouy. Sur la constitution de la charge electrique a la surface d'un electrolyte. J PHYS THEOR APPL, 9(1):457-468, 1910.

[120] C.H. Hamann, A. Hamnett, and W. Vielstich. Electrochemistry. Wiley, 2007.

[121] M.Z. Bazant, M.S. Kilic, B. Storey, and A. Ajdari. Towards an understanding of induced-charge electrokinetics at large applied voltages in concentrated solutions. ADV COLLOID INTERFAC, 152:48-88, 2009 .

[122] P.M. Biesheuvel, M. van Soestbergenb, and M.Z. Bazant. Imposed currents in galvanic cells. ELECTROCHIM ACTA, 54:4857-4871, 2009.

[123] J. Marcicki, A.T. Conlisk, and G. Rizzoni. A Lithium-ion battery model including electrical double layer effects. J POWER SOURCES, 125:157-169, 2014.

[124] M.Z. Bazant, K. Thornton, and A. Ajdari. Diffuse-charge dynamics in electrochemical systems. PHYS REV E, 70:021506, 2004.

[125] A. Frumkin, O. Petry, and B. Damaskin. The notion of the electrode charge and the Lippmann equation. J ELECTROANAL CHEM, 27(1):81 - 100, 1970.

[126] J.M. Rubi and S. Kjelstrup. Mesoscopic nonequilibrium thermodynamics gives the same thermodynamic basis to Butler-Volmer and Nernst equations. J PHYS CHEM B, 107:13471-13477, 2003.

[127] A.F. Bower and P.M. Guduru. A simple finite element model of diffusion, finite deformation, plasticity and fracture in Lithium ion insertion electrode materials. Modelling Simul. Mater. Sci. Eng., 20:045004, 2012 . 
[128] G. Bucci, S.P.V. Nadimpalli, V.A. Sethuraman, A.F. Bower, and P.R. Guduru. Measurement and modeling of the mechanical and electrochemical response of amorphous Si thin film electrodes during cyclic lithiation. J MECH PHYS SOLIDS, 62:276-294, 2014.

[129] T.S. Dao, C.P. Vyasarayani, and J. McPhee. Simplification and order reduction of Lithium-ion battery model based on porous-electrode theory. J POWER SOURCES, 198:329-337, 2012.

[130] I. Streeter and R.G. Compton. Numerical simulation of potential step chronoamperometry at low concentrations of supporting electrolyte. J PHYS CHEM-US, 112:13716-13728, 2008.

[131] W. Dreyer, C. Guhlke, and M. Landstorfer. A mixture theory of electrolytes containing solvation effects. ELECTROCHEM COMMUN, 43:75-78, 2014.

[132] A. Gillman, G. Amadio, K. Matouš, and T.L. Jackson. Third-order thermo-mechanical properties for packs of platonic solids using statistical micromechanics. P R SOC A, 471, 2015.

[133] Y.T. Cheng and M.W. Verbrugge. Evolution of stress within a spherical insertion electrode particle under potentiostatic and galvanostatic operation. J POWER SOURCES, 190:453-460, 2009.

[134] Y.T. Cheng and M.W. Verbrugge. Diffusion-induced stress, interfacial charge transfer, and criteria for avoiding crack initiation of electrode particles. J ELECTROCHEM SOC, 4:508-516, 2010.

[135] J. Christensen and J. Newman. Stress generation and fracture in Lithium insertion materials. J SOLID STATE ELECTR, 10:293-319, 2006.

[136] Z. Cui, F. Gao, and J. Qu. A finite deformation stress-dependent chemical potential and its applications to Lithium ion batteries. J MECH PHYS SOLIDS, 60:1280-1295, 2012.

[137] R. Deshpande, Y.T. Cheng, and M.W. Verbrugge. Modeling diffusion-induced stress in nanowire electrode structures. J POWER SOURCES, 195:5081-5088, 2010.

[138] R. Deshpande, Y.T Cheng, M.W. Verbrugge, and A. Timmons. Diffusion induced stresses and strain energy in a phase-transforming spherical electrode particle. J ELECTROCHEM SOC, 158(6):A718A724, 2011.

[139] S. Golmon, K. Maute, S.H. Lee, and M.L. Dunn. Stress generation in silicon particles during Lithium insertion. APPL PHYS LETT, 97:033111, 2010.

[140] C. Miehe and H. Dal. Computational electro-chemo-mechanics of Lithium-ion battery electrodes at finite strains. COMPUT MECH, 55:303-325, 2015.

[141] R.T. Purkayastha and R.M. McMeeking. A parameter study of intercalation of Lithium into storage particles in a Lithium-ion battery. COMP MATER SCI, 80:2-14, 2013.

[142] X. Zhang, A.M. Sastry, and W. Shyy. Intercalation-induced stress and heat generation within single Lithium-ion battery chatode particles. J ELECTROCHEM SOC, 155(7):A542-A552, 2008.

[143] X. Zhang, W. Shyy, and A.M. Sastry. Numerical simulation of intercalation-induced stress in Li-ion battery electrode particles. J ELECTROCHEM SOC, 154:A910-A916, 2007.

[144] K. Zhao, M. Pharr, S. Cai, J.J. Vlassak, and Z. Suo. Large plastic deformation in high-capacity Lithium-ion batteries caused by charge and discharge. J AM CERAM SOC, 94(S1):S226-S235, 2011.

[145] A. Awarke, S. Lauer, M. Wittler, and S. Pischinger. Quantifying the effects of strains on the conductivity and porosity of $\mathrm{LiFePO}_{4}$ based Li-ion composite cathodes using a multi-scale approach. COMP MATER SCI, 50(3):871-879, 2011.

[146] A. Gupta, J. H. Seo, X. Zhang, W. Du, A.M. Sastry, and W. Shyy. Effective transport properties of $\mathrm{LiMn}_{2} \mathrm{O}_{4}$ electrode via particle-scale modeling. J ELECTROCHEM SOC, 158(5):A487-A497, 2011.

[147] S.A. Roberts, V.E. Brunini, K.N. Long, and A.M. Grillet. A framework for three-dimensional mesoscale modeling of anisotropic swelling and mechanical deformation in Lithium-ion electrodes. J ELECTROCHEM SOC, 161(11):F3052-F3059, 2014. 
[148] A.J. Stershic, S. Simunovic, and J. Nanda. Modeling the evolution of Lithium-ion particle contact distributions using a fabric tensor approach. J POWER SOURCES, 297:540-550, 2015.

[149] M. Ender, J. Joos, T. Carraro, and E. Ivers-Tiffee. Three dimensional reconstruction of a composite cathode for Lithium-ion cells. ELECTROCHEM COMMUN, 13(2):166-168, 2011.

[150] T. Hutzenlaub, S. Thiele, R. Zengerle, and C. Ziegler. Three-dimensional reconstruction of a $\mathrm{LiCoO}_{2}$ Li-ion battery cathode. ELECTROCHEM SOLID ST, 15(3):A33-A36, 2012.

[151] T. Hutzenlaub, S. Thiele, R. Paust, R.M. Spotnitz, R. Zengerle, and C. Walchshofer. Three dimensional electrochemical Li-ion battery modeling featuring a focused ion-beam/scanning electrode microscopy based three-phase reconstruction of a $\mathrm{LiCoO}_{2}$ cathode. ELECTROCHIM ACTA, 115:131-139, 2014.

[152] V. Malave, J. Berger, H. Zhu, and R.J. Kee. A computational model of the mechanical behavior within reconstructed $\mathrm{Li}_{x} \mathrm{CoO}_{2}$ Li-ion battery cathode particles. ELECTROCHIM ACTA, 130:707-717, 2014.

[153] V. Malave, J.R. Berger, and P.A. Martin. Concentration-dependent chemical expansion in Lithium-ion battery cathode particles. J APPL MECH, 81(9), 2014.

[154] D.E. Stephenson, E.M. Hartman, J.N. Harb, and D.R. Wheeler. Modeling of particle-particle interactions in porous cathodes for Lithium-ion batteries. J ELECTROCHEM SOC, 154:A1146-A1155, 2007.

[155] Christian Wieser, Torben Prill, and Katja Schladitz. Multiscale simulation process and application to additives in porous composite battery electrodes. J POWER SOURCES, 277:64-75, 2015.

[156] S.K. Babu, A.I. Mohamed, J.F. Whitacre, and S. Litster. Multiple imaging mode X-ray computed tomography for distinguishing active and inactive phases in Lithium-ion battery cathodes. J POWER SOURCES, 283:314-319, 2015.

[157] P.R. Shearing, L.E. Howard, P. Jorgensen, N.P. Brandon, and S.J. Harris. Characterization of the 3dimensional microstructure of a graphite negative electrode from a Li-ion battery. ELECTROCHEM COMMUN, 12(3):374 - 377, 2010.

[158] L. Zielke, T. Hutzenlaub, D.R. Wheeler, I. Manke, T. Arlt, N. Paust, R. Zengerle, and S. Thiele. A combination of X-Ray tomography and carbon binder modeling: reconstructing the three phases of $\mathrm{LiCoO}_{2}$ Li-ion battery cathodes. ADV ENERGY MATER, 4(8):1301617, 2014.

[159] L. Zielke, T. Hutzenlaub, D.R. Wheeler, C.W. Chao, I. Manke, A. Hilger, N. Paust, R. Zengerle, and S. Thiele. Three-phase multiscale modeling of a $\mathrm{LiCoO}_{2}$ cathode: combining the advantages of FIB-SEM imaging and X-Ray tomography. ADV ENERGY MATER, 5:1401612, 2015.

[160] J.R. Wilson, J.S. Cronin, S.A. Barnett, and S.J. Harris. Measurements of three-dimensional microstructure in a $\mathrm{LiCoO}_{2}$ positive electrode. J POWER SOURCES, 196:3443-3447, 2011.

[161] Z. Liu, J.S. Cronin, Y. Chen-Wiegart, J.R. Wilson, K.J. Yakal-Kremski, J. Wang, K.T. Faber, and S.A. Barnett. Three-dimensional morphological measurements of $\mathrm{LiCoO}_{2}$ and $\mathrm{LiCoO}_{2} / \mathrm{Li}\left(\mathrm{Ni}_{1 / 3} \mathrm{Mn}_{1 / 3} \mathrm{Co}_{1 / 3}\right) \mathrm{O}_{2}$ Lithium-ion battery cathodes. J POWER SOURCES, 227:267-274, 2013.

[162] A.H. Wiedemann, G.M. Goldin, S.A. Barnett, H. Zhu, and R.J. Kee. Effects of three-dimensional cathode microstructure on the performance of Lithium-ion battery cathodes. ELECTROCHIM ACTA, pages $580-588,2013$.

[163] Z. Liu, Y. Chen-Wiegart, J. Wang, S.A. Barnett, and K.T. Faber. Three-phase 3d reconstructions of a $\mathrm{LiCoO}_{2}$ cathode via FIB-SEM tomography. MICROSC MICROANAL, 22:140-148, 2016.

[164] J. Newman and W.M. Tiedemann. Temperature rise in a battery module with constant heat generation. J ELECTROCHEM SOC, 142(4):1054-1057, 1995.

[165] C.R. Pals and J. Newman. Thermal modeling of the Lithium/polymer battery. J ELECTROCHEM SOC, 142(10):3274-3281, 1995. 
[166] L. Rao and J. Newman. Heat-generation rate and general energy balance for insertion battery systems. J ELECTROCHEM SOC, 144(8):2697-2704, 1997.

[167] C.Y. Jhua, Y.W. Wang, C.Y. Wen, and C.M. Shu. Thermal runaway potential of $\mathrm{LiCoO}_{2}$ and $\mathrm{Li}\left(\mathrm{Ni}_{1 / 3} \mathrm{Co}_{1 / 3} \mathrm{Mn}_{1 / 3}\right) \mathrm{O}_{2}$ batteries determined with adiabatic calorimetry methodology. APPL ENERG, 100(127-131), 2012.

[168] S. Abada, G. Marlair, A. Lecocq, M. Petit, V. Sauvant-Moynot, and F. Huet. Safety focused modeling of Lithium-ion batteries: A review. J POWER SOURCES, 306:178 - 192, 2016.

[169] M. Fleckenstein, O. Bohlen, M.A. Roscher, and B. Baeker. Current density and state of charge inhomogeneities in Li-ion battery cells with $\mathrm{LiFePO}_{4}$ as cathode material due to temperature gradients. J POWER SOURCES, 196:4769-4778, 2011.

[170] A. Latz and J. Zausch. Thermodynamic consistent transport theory of Li-ion batteries. J POWER SOURCES, 196:3296-3302, 2011.

[171] S. Rothe, J.H. Schmidt, and S. Hartmann. Analytical and numerical treatment of electro-thermomechanical coupling. ARCH APPL MECH, 85:1245-1264, 2015.

[172] I. Baghdadi, O. Briat, J.Y. Delétage, P. Gyan, and J.M. Vinassa. Lithium battery aging model based on Dakin's degradation approach. J POWER SOURCES, 325:273 - 285, 2016.

[173] R. Darling and J. Newman. Modeling side reactions in composite $\mathrm{Li}_{\mathrm{y}} \mathrm{Mn}_{2} \mathrm{O}_{4}$ electrodes. $\underline{\mathrm{J}}$ ELECTROCHEM SOC, 145(3):990-998, 1998.

[174] J.R. Belt, C.D. Ho, C.G. Motloch, T.J. Miller, and T.Q. Duong. A capacity and power fade study of Li-ion cells during life cycle testing. J POWER SOURCES, 123(2):241-246, 2003.

[175] I. Bloom, B.W. Cole, J.J. Sohn, S.A. Jones, E.G. Polzin, V.S. Battaglia, G.L. Henriksen, C. Motloch, R. Richardson, T. Unkelhaeuser, D. Ingersoll, and H.L. Case. An accelerated calendar and cycle life study of Li-ion cells. J POWER SOURCES, 101(2):238-247, 2001.

[176] K. Asakura, M. Shimomura, and T. Shodai. Study of life evaluation methods for Li-ion batteries for backup applications. J POWER SOURCES, 119-121:902-905, 2003. Selected papers presented at the 11th International Meeting on Lithium Batteries.

[177] M. Safari and C. Delacourt. Simulation-based analysis of aging phenomena in a commercial graphite/LiFePO 4 cell. J ELECTROCHEM SOC, 158(12):A1436-A1447, 2011.

[178] Y.P. Wu, E. Rahm, and R. Holze. Carbon anode materials for Lithium ion batteries. J POWER SOURCES, 114(2):228-236, 2003.

[179] R. Fong, U. von Sacken, and J. R. Dahn. Studies of Lithium intercalation into carbons using nonaqueous electrochemical cells. J ELECTROCHEM SOC, 137(7):2009-2013, 1990.

[180] J. Christensen and J. Newman. A mathematical model for the Lithium-ion negative electrode solid electrolyte interphase. J ELECTROCHEM SOC, 151(11):A1977-A1988, 2004.

[181] J. Deng, G.J. Wagner, and R.P. Muller. Phase field modeling of solid electrolyte interface formation in Lithium ion batteries. J ELECTROCHEM SOC, 160(3):A487-A496, 2013.

[182] D. Li, D. Danilov, Z. Zhang, H. Chen, Y. Yang, and P.H.L Notten. Modeling the SEI-formation on graphite electrodes in $\mathrm{LiFePO}_{4}$ batteries. J ELECTROCHEM SOC, 162(6):A858-A869, 2015.

[183] L. Liu, J. Park, X. Lin, A.M. Sastry, and W. Lu. A thermal-electrochemical model that gives spatialdependent growth of solid electrolyte interphase in a Li-ion battery. J POWER SOURCES, 268:482490, 2014.

[184] M. Nie, D. Chalasani, D.P. Abraham, Y. Chen, A. Bose, and B.L. Lucht. Lithium ion battery graphite solid electrolyte interphase revealed by microscopy and spectroscopy. J PHYS CHEM-US, 117:1257$1267,2013$. 
[185] H.J. Ploehn, P. Ramadass, and R.E. White. Solvent diffusion model for aging of Lithium-ion battery cells. J ELECTROCHEM SOC, 151(3):A456-A462, 2004.

[186] E. Rejovitzky, C.V. Di Leo, and L. Anand. A theory and a simulation capability for the growth of a solid electrolyte interphase layer at an anode particle in a Li-ion battery. J MECH PHYS SOLIDS, 78(210-230), 2015.

[187] H. Shin, J. Park, S. Han, A.M. Sastry, and W. Lu. Component-/structure-dependent elasticity of solid electrolyte interphase layer in Li-ion batteries: Experimental and computational studies. J POWER SOURCES, 277:169-179, 2015.

[188] H. Shin, J. Park, A.M. Sastry, and W. Lu. Degradation of the solid electrolyte interphase induced by the deposition of manganese ions. J POWER SOURCES, 284:416-427, 2015.

[189] M. Tang, S. Lu, and J. Newman. Experimental and theoretical investigation of solid-electrolyteinterphase formation mechanisms on glassy carbon. J ELECTROCHEM SOC, 159(11):A1775-A1785, 2012 .

[190] Y. Xie, J. Li, and C. Yuan. Multiphysics modeling of Lithium ion battery capacity fading process with solid-electrolyte interphase growth by elementary reaction kinetics. J POWER SOURCES, 248:172$179,2014$.

[191] H. Ekström and G. Lindbergh. A model for predicting capacity fade due to SEI formation in a commercial graphite/LiFePO 4 cell. J ELECTROCHEM SOC, 162(6):A1003-A1007, 2015.

[192] D. Bothe. On the Maxwell-Stefan approach to multicomponent diffusion. PROG NONLIN, 80:81-93, 2011.

[193] S.H. Lam. Multicomponent diffusion revisited. PHYSICS OF FLUIDS, 18:073101, 2006.

[194] Y.T. Cheng and M.W. Verbrugge. The influence of surface mechanics on diffusion induced stresses within spherical nanoparticles. J APPL PHYS, 104:083521, 2008.

[195] K. Higa and V. Srinivasan. Stress and strain in silicon electrode models. J ELECTROCHEM SOC, 162(6):A1111-A1122, 2015.

[196] R.A. Huggins and W.D. Nix. Decrepitation model for capacity loss during cycling of alloys in rechargeable electrochemical systems. IONICS, 6:57-63, 2000.

[197] K. Zhao, M. Pharr, S. Cai, J.J. Vlassak, and Z. Suo. Fracture of electrodes in Lithium-ion batteries caused fast charging. J APPL PHYS, 108:073517, 2010.

[198] K.E. Aifantis and J.P. Dempsey. Stable crack growth in nanostructured Li-batteries. J POWER SOURCES, 143:203-211, 2005.

[199] K.E. Aifantis, S.A. Hackney, and J.P. Dempsey. Design criteria for nanostructured Li-ion batteries. J POWER SOURCES, 165:874-879, 2007.

[200] Y. Hu, X. Zhao, and Z. Suo. Averting cracks caused by insertion reaction in Lithium-ion batteries. J MATER RES, 25(6):1007-1010, 2010.

[201] I. Ryu, J. W. Choi, Y. Cui, and W.D. Nix. Size-dependent fracture of si nanowire battery anodes. J MECH PHYS SOLIDS, 59:1717-1730, 2011.

[202] H. Woodford, Y.M. Chiang, and W.C. Carter. Electrochemical shock of intercalation electrodes: A fracture mechanics analysis. J ELECTROCHEM SOC, 157(10):A1052-A1059, 2010.

[203] K. Zhao, M. Pharr, J.J. Vlassak, and Z. Suo. Inelastic hosts as electrodes for high-capacity Lithium-ion batteries. J APPL PHYS, 109:016110, 2011.

[204] A. Salvadori. A plasticity framework for (linear elastic) fracture mechanics. J MECH PHYS SOLIDS, 56:2092-2116, 2008. 
[205] A. Salvadori. Crack kinking in brittle materials. J MECH PHYS SOLIDS, 58:1835-1846, 2010.

[206] A. Salvadori and F. Fantoni. Minimum theorems in 3D incremental linear elastic fracture mechanics. INT J FRACTURE, 184(1):57-74, 2013.

[207] A. Salvadori and F. Fantoni. Fracture propagation in brittle materials as a standard dissipative process: general theorems and crack tracking algorithms. J MECH PHYS SOLIDS, in press, 2016.

[208] A. Salvadori and A. Giacomini. The most dangerous flaw orientation in brittle materials and structures. INT J FRACTURE, 183(1):19-28, 2013. DOI: 10.1007/s10704-013-9872-x.

[209] T.K. Bhandakkar and H. Gao. Cohesive modeling of crack nucleation under diffusion induced stresses in a thin strip: Implications on the critical size for flaw tolerant battery electrodes. INT J SOLIDS STRUCT, 47:1424-1434, 2010.

[210] T.K. Bhandakkar and H. Gao. Cohesive modeling of crack nucleation in a cylindrical electrode under axisymmetric diffusion induced stresses. INT J SOLIDS STRUCT, 48:2304-2309, 2011.

[211] C. Miehe, L.M. Schaenzel, and H. Ulmer. Phase field modeling of fracture in multi-physics problems. Part I. balance of crack surface and failure criteria for brittle crack propagation in thermo-elastic solids. COMPUT METHOD APPL M, 294:449-485, 2015.

[212] C. Miehe, M. Hofacker, L.M. Schaenzel, and F. Aldakheel. Phase field modeling of fracture in multiphysics problems. Part II. coupled brittle-to-ductile failure criteria and crack propagation in thermoelastic-plastic solids. COMPUT METHOD APPL M, 294:486-522, 2015.

[213] C. Miehe, H. Dal, and A. Raina. A phase field model for chemo-mechanical induced fracture in Lithium-ion battery electrode particles. INT J NUMER METH ENG, 106(9):683-711, 2016.

[214] P. Zuo and Y.P. Zhao. A phase field model coupling Lithium diffusion and stress evolution with crack propagation and application in Lithium ion batteries. PHYS CHEM CHEM PHYS, 17:287-297, 2015.

[215] C. Miehe and S. Mauthe. Phase field modeling of fracture in multi-physics problems. Part III. crack driving forces in hydro-poro-elasticity and hydraulic fracturing of fluid-saturated porous media. COMPUT METHOD APPL M, 304:619-655, 2016.

[216] Y. Zhao, B.X. Xu, P. Stein, and D. Gross. Phase-field study of electrochemical reactions at exterior and interior interfaces in Li-ion battery electrode particles. COMPUT METHOD APPL M, pages -, 2016.

[217] M. Klinsmann, D. Rosato, M. Kamlah, and R.M. McMeeking. Modeling crack growth during Li extraction in storage particles using a fracture phase field approach. J ELECTROCHEM SOC, 163(2):A102A118, 2016.

[218] D.T. O'Connor, M.J. Welland, W.K. Liu, and P.V. Voorhees. Phase transformation and fracture in single $\mathrm{Li}_{x} \mathrm{FePO}_{4}$ cathode particles: a phase-field approach to Li-ion intercalation and fracture. MODELLING SIMUL MATER SCI ENG, 24(3):035020, 2016.

[219] M. Klinsmann, D. Rosato, M. Kamlah, and R.M. McMeeking. Modeling crack growth during Li insertion in storage particles using a fracture phase field approach. J MECH PHYS SOLIDS, 92:313 $344,2016$.

[220] J. Christensen and J. Newman. A mathematical model of stress generation and fracture in Lithium manganese oxide. J ELECTROCHEM SOC, 153(6):A1019-A1030, 2006.

[221] M.W. Verbrugge and Y.T. Cheng. Stress and strain-energy distributions within diffusion- controlled insertion-electrode particles subjected to periodic potential excitations. J ELECTROCHEM SOC, 156:A927-A937, 2009.

[222] Y.F. Gao and M. Zhou. Strong stress-enhanced diffusion in amorphus Lithium alloy nanowire electrodes. J APPL PHYS, 109:014310, 2011. 
[223] J.C.M. Li. Physical chemistry of some microstructural phenomena. METALL TRANS, 9A:1353-1380, 1978.

[224] S. Prussin. Generation and distribution of dislocations by solute diffusion. J APPL PHYS, 32(10):1876$1881,1961$.

[225] S. Lee, W.L. Wang, and J.R. Chen. Diffusion-induced stresses in a hollow cylinder: Constant surface stresses. MATER CHEM PHYS, 64(2):123 - 130, 2000.

[226] F. Yang. Interaction between diffusion and chemical stresses. MATER SCI ENG A, 409:153-159, 2005.

[227] F. Larche and J.W. Cahn. A linear theory of thermochemical equilibrium under stress. ACTA METALL MATER, 21:1051-1063, 1973.

[228] F. Larche and J.W. Cahn. Non linear theory of thermochemical equilibrium under stress. ACTA METALL MATER, 26:53-60, 1978.

[229] R.T. Purkayastha and R.M. McMeeking. A linearized model for Lithium ion batteries and maps for their performance and failure. J APPL MECH, 79:1-16, 2012.

[230] J.H. Seo, M. Chung, M. Park, S.W. Han, X. Zhang, and A.M. Sastry. Generation of realistic structures and simulations of internal stress: A numerical/AFM study of $\mathrm{LiMn}_{2} \mathrm{O}_{4}$ particles. J ELECTROCHEM SOC, 158(4):A434-A442, 2011.

[231] C.H. Wu. The role of Eshelby stress in composition-generated and stress-assisted diffusion. J MECH PHYS SOLIDS, 49(8):1771-1794, 2001.

[232] H. Haftbaradaran, J. Song, W.A. Curtin, and H. Gao. Continuum and atomistic models of strongly coupled diffusion, stress, and solute concentration. J POWER SOURCES, 196:361-370, 2011.

[233] J. W. Wang, Y. He, F. Fan, X.H. Liu, S. Xia, Y. Liu, C.T. Harris, H. Li, J.Y. Huang, S.X. Mao, and T. Zhu. Two-phase electrochemical lithiation in amorphous silicon. NANO LETT, 13(2):709-715, 2013.

[234] L. Brassart, K. Zhao, and Z. Suo. Cyclic plasticity and shakedown in high-capacity electrodes of Lithium-ion batteries. INT J SOLIDS STRUCT, 50:1120-1129, 2013.

[235] V.A. Sethuraman, V. Srinivasan, A. F. Bower, and P.R. Guduru. In situ measurements of stresspotential coupling in lithiated silicon. J ELECTROCHEM SOC, 157:1253-1261, 2010.

[236] J. Wang, Y. Chen-Wiegart, and J. Wang. In operando tracking phase transformation evolution of Lithium iron phosphate with hard X-ray microscopy. NAT COMMUN, 5, 2014.

[237] G. Chen, X. Song, and T.J. Richardson. Electron microscopy study of the $\mathrm{LiFePO}_{4}$ to $\mathrm{FePO}_{4}$ phase transition. ELECTROCHEM SOLID ST, 9:A295, 2006.

[238] L. Laffont, C. Delacourt, P. Gibot, M. Wu, P. Kooyman, C. Masquelier, and J. Tarascon. Study of the $\mathrm{LiFePO}_{4} / \mathrm{FePO}_{4}$ two-phase system by high-resolution electron energy loss spectroscopy. CHEM MATER, 18(23):5520-5529, 2006.

[239] J.W. Cahn and J. E. Hilliard. Free energy of a nonuniform system. I. interfacial free energy. J CHEM PHYS, 28:258-267, 1958.

[240] K. Thornton, J. Agren, and P.W. Voorhees. Modelling the evolution of phase boundaries in solids at the meso- and nano-scales. ACTA MATER, 51(19):5675 - 5710, 2003.

[241] V. Srinivasan and J. Newman. Discharge model for the Lithium iron-phosphate electrode. J ELECTROCHEM SOC, 151(10):A1517-A1529, 2004.

[242] V. R. Subramanian, H. J. Ploehn, and R. E. White. Shrinking core model for the discharge of a metal hydride electrode. J ELECTROCHEM SOC, 147(8):2868-2873, 2000. 
[243] Q. Zhang and R.E. White. Moving boundary model for the discharge of a $\mathrm{LiCoO}_{2}$ electrode. $\underline{\mathrm{J}}$ ELECTROCHEM SOC, 154(6):A587-A596, 2007.

[244] F. Gao and W. Hong. Phase-field model for the two-phase lithiation of silicon. J MECH PHYS SOLIDS, 94:18 - 32, 2016.

[245] G.K. Singh, G. Ceder, and M.Z. Bazant. Intercalation dynamics in rechargeable battery materials: General theory and phase-transformation waves in $\mathrm{LiFePO}_{4}$. ELECTROCHIM ACTA, 53:7599-7613, 2008.

[246] D. Burch, G.K. Singh, G. Ceder, and M.Z. Bazant. Phase-transformation wave dynamics in $\mathrm{LiFePO}_{4}$. Solid State Phenomena, 139:95-100, 2008.

[247] B.C. Han, A. Van der Ven, D. Morgan, and G. Ceder. Electrochemical modeling of intercalation processes with phase field models. ELECTROCHIM ACTA, 49(26):4691 - 4699, 2004.

[248] M. Tang, W. Craig Carter, J.F. Belak, and Y.M. Chiang. Modeling the competing phase transition pathways in nanoscale olivine electrodes. ELECTROCHIM ACTA, 56(2):969 - 976, 2010.

[249] M. Tang, H.-Y. Huang, N. Meethong, Y.-H. Kao, W. C. Carter, and Y.-M. Chiang. Model for the particle size, overpotential, and strain dependence of phase transition pathways in storage electrodes: Application to nanoscale olivines. CHEM MATER, 21(8):1557-1571, 2009.

[250] D.A. Cogswell and M.Z. Bazant. Coherency strain and the kinetics of phase separation in $\mathrm{LiFePO}_{4}$ nanoparticles. ACS NANO, 6(3):2215-2225, 2012.

[251] L. Anand. A Cahn-Hilliard-type theory for species diffusion coupled with large elastic-plastic deformations. J MECH PHYS SOLIDS, 60(12):1983-2002, 2012.

[252] C. Di Leo, E. Rejovitzky, and L. Anand. A Cahn-Hilliard-type phase-field theory for species diffusion coupled with large elastic deformations: Application to phase-separating Li-ion electrode materials. $\mathrm{J}$ MECH PHYS SOLIDS, 70:1-29, 2014.

[253] P. Areias, E. Samaniego, and T. Rabczuk. A staggered approach for the coupling of Cahn-Hilliard type diffusion and finite strain elasticity. COMPUT MECH, 57(2):339-351, 2016.

[254] S. Huang, F. Fan, J. Li, S. Zhang, and T. Zhu. Stress generation during lithiation of high-capacity electrode particles in Lithium ion batteries. ACTA MATER, 61:4354-4364, 2013.

[255] H. Yang, F. Fan, W. Liang, X. Guo, T. Zhu, and S. Zhang. A chemo-mechanical model of lithiation in silicon. J MECH PHYS SOLIDS, 70:349-361, 2014.

[256] A.D. Drozdov. A model for the mechanical response of electrode particles induced by Lithium diffusion in Li-ion batteries. ACTA MECH, 225:2987-3005, 2014.

[257] A.D. Drozdov. Viscoplastic response of electrode particles in Li-ion batteries driven by insertion of Lithium. INT J SOLIDS STRUCT, 51:690-705, 2014.

[258] Z. Cui, F. Gao, and J. Qu. Interface-reaction controlled diffusion in binary solids with applications to lithiation of silicon in Lithium ion batteries. J MECH PHYS SOLIDS, 61:293-310, 2013.

[259] K. Zhao, M. Pharr, Q. Wan, W.L. Wang, E. Kaxiras, J.J. Vlassak, and Z. Suo. Concurrent reaction and plasticity during initial lithiation of crystalline silicon in Lithium-ion batteries. J ELECTROCHEM SOC, 159:A238-A243, 2012.

[260] H. Yang, S. Huang, X. Huang, F. Fan, W. Liang, X.H. Liu, L.Q. Chen, J.Y. Huang, J. Li, T. Zhu, and S. Zhang. Orientation-dependent interfacial mobility governs the anisotropic swelling in lithiated silicon nanowires. NANO LETT, 12(4):1953-1958, 2012.

[261] W.J. Drugan and J.R. Willis. A micromechanics-based nonlocal constitutive equation and estimates of representative volume element size for elastic composites. J MECH PHYS SOLIDS, 44(4):497 - 524, 1996. 
[262] Z. Shan and A. M. Gokhale. Representative volume element for non-uniform micro-structure. COMP MATER SCI, 24(3):361 - 379, 2002.

[263] S. Swaminathan, S. Ghosh, and N. J. Pagano. Statistically equivalent representative volume elements for unidirectional composite microstructures: Part I - without damage. J COMPOS MATER, 40(7):583-604, 2006.

[264] S. Torquato. Random Heterogeneous Materials: Microstructure and Macroscopic Properties. Springer, 2002.

[265] J.R. Willis. Elasticity Theory of Composites. Defense Technical Information Center, 1980.

[266] D. E. Stephenson, B. C. Walker, C. B. Skelton, E. P. Gorzkowski, D J. Rowenhorst, and D. R. Wheeler. Modeling 3D microstructure and ion transport in porous Li-ion battery electrodes. J ELECTROCHEM SOC, 158:A781-A789, 2011.

[267] T.R. Ferguson and M.Z. Bazant. Nonequilibrium thermodynamics of porous electrodes. $\underline{J}$ ELECTROCHEM SOC, 159(12):A1967-A1985, 2012.

[268] M. Schmuck and M.Z. Bazant. Homogenization of the Poisson-Nernst-Planck equations for ion transport in charged porous media. SIAM J APPL MATH, 75(3):1369-1401, 2015.

[269] S. Lee, A.M. Sastry, and J. Park. Study on microstructures of electrodes in Lithium-ion batteries using variational multi-scale enrichment. J POWER SOURCES, 315:96 - 110, 2016.

[270] S. Golmon, K. Maute, and M.L. Dunn. Numerical modeling of electrochemical-mechanical interactions in Lithium polymer batteries. Computer and Structures, 87:1567-1579, 2009.

[271] S. Golmon, K. Maute, and M.L. Dunn. Multiscale design optimization of Lithium ion batteries using adjoint sensitivity analysis. INT J NUMER METH ENG, 92:475-494, 2012.

[272] S. Golmon, K. Maute, and M.L. Dunn. A design optimization methodology for $\mathrm{Li}^{+}$batteries. J POWER SOURCES, 253:239-250, 2014.

[273] S. Allu, S. Kalnaus, S. Simunovic, J. Nanda, J.A. Turner, and S. Pannala. A three-dimensional mesomacroscopic model for Li-ion intercalation batteries. J POWER SOURCES, 325:42 - 50, 2016.

[274] P.M. Suquet. Local and global aspects in the mathematical theory of plasticity. In A. Sawczuk and G. Bianchi, editors, Plasticity today: modeling, methods and applications, pages 279-310. Elsevier Applied Science Publishers, London, 1985.

[275] M.G.D. Geers, V.G. Kouznetsova, and Brekelmans W.A.M. Multi-scale computational homogenization: trends and challenges. J COMPUT APPL MATH, 234:2175-2182, 2010.

[276] A. Kovetz. The principles of electromagnetic theory. Cambridge University Press, 1989.

[277] R.A. Huggins. Energy storage. Springer, 2010.

[278] A.A. Franco, M.L. Doublet, and W.G.B. Bessler, editors. Physical Multiscale Modeling and Numerical Symulation of Electrochemical Devices for Energy Conversion and Storage. Springer, London, 2016.

[279] M.A. Rahman, S. Anwar, and A. Izadian. Electrochemical model parameter identification of a Lithiumion battery using particle swarm optimization method. J POWER SOURCES, 307:86 - 97, 2016.

[280] K. Takahashi, K. Higa, S. Mair, M. Chintapalli, N. Balsara, and V. Srinivasan. Mechanical degradation of graphite/PVDF composite electrodes: A model-experimental study. J ELECTROCHEM SOC, 163(3):A385-A395, 2016. 